\title{
Semi-invariants of symmetric quivers of tame type
}

\author{
Riccardo Aragona \\ Università degli Studi di Roma "Tor Vergata" \\ Dipartimento di Matematica \\ Via della Ricerca Scientifica 1, 00133 Rome (Italy) \\ E-mail: ric_aragona@yahoo.it
}

\begin{abstract}
A symmetric quiver $(Q, \sigma)$ is a finite quiver without oriented cycles $Q=\left(Q_{0}, Q_{1}\right)$ equipped with a contravariant involution $\sigma$ on $Q_{0} \sqcup Q_{1}$. The involution allows us to define a nondegenerate bilinear form $<,>$ on a representation $V$ of $Q$. We shall say that $V$ is orthogonal if $<,>$ is symmetric and symplectic if $<,>$ is skew-symmetric. Moreover, we define an action of products of classical groups on the space of orthogonal representations and on the space of symplectic representations. So we prove that if $(Q, \sigma)$ is a symmetric quiver of tame type then the rings of semi-invariants for this action are spanned by the semi-invariants of determinantal type $c^{V}$ and, when matrix defining $c^{V}$ is skew-symmetric, by the Pfaffians $p f^{V}$. To prove it, moreover, we describe the symplectic and orthogonal generic decomposition of a symmetric dimension vector.
\end{abstract}

Keywords: Representations of quivers; Invariants; Classical groups; Coxeter functors; Pfaffian; Schur modules; Generic decomposition.

\section{Introduction}

The symmetric quivers and their orthogonal and symplectic representations have been introduced by Derksen and Weyman in [8] to provide a formalization in the quiver setting of some problem related to representations of classical groups. Generalizations of quivers and their representations have been defined in a different setting by Zubkov in [24] and Shmelkin in [22].

The representations of symmetric quivers could be a tool to classify products of flag varieties with finitely many orbits under the diagonal action of classical groups. Magyar, Weyman and Zelevinsky in [16] solved this problem for general linear groups using usual quiver setting.

It would be also interesting to generalize to symmetric quivers the results about virtual representations and virtual semi-invariants of quivers given by Igusa, Orr, Todorov and Weyman in [11].

The author, in [3], displayed a set of generators of rings of semi-invariants of symmetric quivers of finite type. In this paper, the second one extract from his PhD thesis, supervised by Professor Jerzy Weyman, we provide similar results for symmetric quivers of tame type. Similar problems, in a different setting, have been studied by Lopatin in [13], using ideas from [14].

Analogous results for usual quivers have been obtained independently by Derksen and Weyman in [7], Domokos and Zubkov in [9] and Schofield and Van den Bergh in [21].

Let $Q=\left(Q_{0}, Q_{1}\right)$ be a quiver, where $Q_{0}$ and $Q_{1}$ are respectively the set of vertices and the set of arrows of $Q$, and let $\sigma$ be an involution on $Q_{0} \sqcup Q_{1}$. The pair $(Q, \sigma)$ is called symmetric quiver.

Let $V$ be a representation of $Q$. The involution allows us to define a nondegenerate bilinear form $<,>$ on $V$. We call the pair $(V,<,>)$ symplectic (respectively orthogonal) representation of $(Q, \sigma)$ if $<$, > is skew-symmetric (respectively symmetric). We define $\operatorname{SpRep}(Q, \beta)$ and $\operatorname{ORep}(Q, \beta)$ to be respectively the space of symplectic $\beta$-dimensional representations and the space of orthogonal $\beta$-dimensional representations of $(Q, \sigma)$. Moreover we can define an action of a product of classical groups, which we call $\operatorname{SSp}(Q, \beta)$ in the symplectic case and 
$S O(Q, \beta)$ in the orthogonal case, on these space. Let $\operatorname{SpSI}(Q, \beta)$ and $\operatorname{SSI}(Q, \beta)$ be respectively the ring of symplectic semi-invariants and the ring of orthogonal semi-invariants of a symmetric quiver $(Q, \sigma)$.

Let $(Q, \sigma)$ be a symmetric quiver and $V$ a representation of the underlying quiver $Q$ such that $\langle\underline{\operatorname{dim}} V, \beta\rangle=0$, where $\langle\cdot, \cdot\rangle$ is the Euler form of $Q$. Let

$$
0 \longrightarrow P_{1} \stackrel{d^{V}}{\longrightarrow} P_{0} \longrightarrow V \longrightarrow 0
$$

be a projective resolution of $V$. We define the semi-invariant $c^{V}:=\operatorname{det}\left(\operatorname{Hom}_{Q}\left(d^{V}, \cdot\right)\right)$ of $\operatorname{SpSI}(Q, \beta)$ and $\operatorname{OSI}(Q, \beta)$ (see [7] and [20]) and, when it is possible, the semi-invariant $p f^{V}:=\operatorname{Pf}\left(\operatorname{Hom}_{Q}\left(d^{V}, \cdot\right)\right)$

Let $C^{+}$be the Coxeter functor and let $\nabla$ be the duality functor. We will prove in the symmetric case the following

Theorem 1. Let $(Q, \sigma)$ be a symmetric quiver of tame type and let $\beta$ be a regular symmetric dimension vector. The ring $\operatorname{SpSI}(Q, \beta)$ is generated by semi-invariants

(i) $c^{V}$ if $V \in \operatorname{Rep}(Q)$ is such that $\langle\underline{\operatorname{dim}} V, \beta\rangle=0$,

(ii) $p f^{V}$ if $V \in \operatorname{Rep}(Q)$ is such that $\langle\underline{\operatorname{dim}} V, \beta\rangle=0, C^{+} V=\nabla V$ and the almost split sequence $0 \rightarrow \nabla V \rightarrow Z \rightarrow$ $V \rightarrow 0$ has the middle term $Z$ in $\operatorname{ORep}(Q)$.

Theorem 2. Let $(Q, \sigma)$ be a symmetric quiver of tame type and let $\beta$ be a regular symmetric dimension vector. The ring $\operatorname{OSI}(Q, \beta)$ is generated by semi-invariants

(i) $c^{V}$ if $V \in \operatorname{Rep}(Q)$ is such that $\langle\underline{\operatorname{dim}} V, \beta\rangle=0$,

(ii) $p f^{V}$ if $V \in \operatorname{Rep}(Q)$ is such that $\langle\underline{\operatorname{dim}} V, \beta\rangle=0, C^{+} V=\nabla V$ and the almost split sequence $0 \rightarrow \nabla V \rightarrow Z \rightarrow$ $V \rightarrow 0$ has the middle term $Z$ in $\operatorname{SpRep}(Q)$.

Differently to the results in [13], our semi-invariants $c^{V}$ and $p f^{V}$ are explicitly related to the representations of $Q$.

The strategy of the proofs is the following. First we set the technique of reflection functors on the symmetric quivers. Then we prove that we can reduce Theorems 1 and 2, by this technique, to particular orientations of the symmetric quivers. Finally, we check Theorems 1 and 2 for these orientations, first for homogeneous regular symmetric dimension vectors $p h$ and then for every other regular symmetric dimension vector.

In the first section we give general notions and results about usual and symmetric quivers and their representations. We state main results 1 and 2 and we recall some results about representations of general linear groups and about invariant theory.

In the second section we adjust to symmetric quivers the technique of reflection functors and we prove general results about semi-invariants of symmetric quivers.

In the third section we check that we can reduce Theorems 1 and 2 to a particular orientation of symmetric quivers of tame type. Then, using classical invariant theory and the technique of Schur functors, we prove Theorems 1 and 2 for symmetric quivers of tame type with this orientation, restricting us to homogeneous regular symmetric dimension vectors. Finally, after having defined and described the symplectic and orthogonal generic decomposition of a symmetric dimension vector (for classical definition see remark 2.8 (a) in [12]), we prove Theorems 1 and 2 for every other regular symmetric dimension vector.

\section{Acknowledgments}

I am deeply grateful to my PhD thesis advisor Jerzy Weyman for his constant guidance and for all suggestions and ideas he shared me. I am grateful to Elisabetta Strickland for the great helpfulness she showed me during the three years of my graduate studies. I also wish to thank Fabio Gavarini and Alessandro D'Andrea for several useful discussions.

\section{Preliminary results}

Throughout the paper $\mathbb{k}$ denotes an algebraically closed field of characteristic 0 . 


\subsection{Representations of quivers}

A quiver $Q$ is a pair $\left(Q_{0}, Q_{1}\right)$ where $Q_{0}$ is a set of vertices, $Q_{1}$ is a set of arrows. For each arrow $a \in Q_{1}$ we shall call $t a \in Q_{0}$ the tail of $a$ and $h a \in Q_{0}$ the head of $a$. Throughout the paper we consider quivers $Q$ without oriented cycles, i.e. in which there are no paths $a_{1} \cdots a_{n}$ such that $t a_{1}=h a_{n}$.

A representation $V$ of $Q$ is a pair $\left\{\{V(x)\}_{x \in Q_{0}},\{V(a): V(t a) \rightarrow V(h a)\}_{a \in Q_{1}}\right\}$ where $V(x)$ is a finite dimensional vector space for every $x \in Q_{0}$ and $V(a)$ is a linear map for every $a \in Q_{1}$. The dimension vector of $V$ is the vector $\underline{\operatorname{dim}}(V):=(\operatorname{dim} V(x))_{x \in Q_{0}} \in \mathbb{N}^{Q_{0}}$.

For a dimension vector $\alpha$ we define $\operatorname{Rep}(Q, \alpha):=\bigoplus_{a \in Q_{0}} \operatorname{Hom}\left(\mathbb{k}^{\alpha(t a)}, \mathbb{k}^{\alpha(h a)}\right)$ the variety of representations of $Q$ of dimension $\alpha$. Moreover we define the action of the group $S L(Q, \alpha)=\prod_{x \in Q_{0}} S L(\alpha(x))<\prod_{x \in Q_{0}} G L(\alpha(x))=$ $G L(Q, \alpha)$ on $\operatorname{Rep}(Q, \alpha)$ by $g \cdot V=\left\{g_{h a} V(a) g_{t a}^{-1}\right\}_{a \in Q_{1}}$ where $g=\left(g_{x}\right)_{x \in Q_{0}} \in G L(Q, \alpha)$ and $V \in \operatorname{Rep}(Q, \alpha)$.

A morphism $f: V \rightarrow W$ of two representations is a family of linear maps $\{f(x): V(x) \rightarrow W(x)\}_{x \in Q_{0}}$ such that $f(h a) V(a)=W(a) f(t a)$ for each $a \in Q_{1}$. Representations and morphisms between representations of a quiver $Q$ define respectively the objects and the morphisms of a category, denoted by $\operatorname{Re} p(Q)$. We denote the space of morphisms from $V$ to $W$ by $\operatorname{Hom}_{Q}(V, W)$ and the space of extensions of $V$ by $W$ by $\operatorname{Ext}_{Q}^{1}(V, W)$.

Finally we define, for every $\alpha, \beta \in \mathbb{Z}^{Q_{0}}$, the non symmetric bilinear form on the space $\mathbb{Z}^{Q_{0}}$ by

$$
\langle\alpha, \beta\rangle=\sum_{x \in Q_{0}} \alpha(x) \beta(x)-\sum_{a \in Q_{1}} \alpha(t a) \beta(h a),
$$

called the Euler form of $Q$.

A vertex $x \in Q_{0}$ is said to be a sink (resp. a source) of $Q$ if $x=h a$ (resp. $x=t a$ ) for every $a \in Q_{1}$ connected to $x$.

Let $x \in Q_{0}$ be a sink (resp. a source) of a quiver $Q$ and let $\left\{a_{1}, \ldots, a_{k}\right\}$ be the arrows connected to $x$. We define the quiver $c_{x}(Q)$ as follows

$$
c_{x}(Q)_{0}=Q_{0} \quad \text { and } \quad c_{x}(Q)_{1}=\left\{c_{x}(a) \mid a \in Q_{1}\right\}
$$

where $t c_{x}\left(a_{i}\right)=h a_{i}, h c_{x}\left(a_{i}\right)=t a_{i}$ for every $i \in\{1, \ldots, k\}$ and $t c_{x}(b)=t b, h c_{x}(b)=h b$ for every $b \in Q_{1} \backslash$ $\left\{a_{1}, \ldots, a_{k}\right\}$. Moreover, we can define the reflection $c_{x}: \mathbb{Z}^{Q_{0}} \rightarrow \mathbb{Z}^{Q_{0}}$ given for $\alpha \in \mathbb{Z}^{Q_{0}}$ by formula

$$
c_{x}(\alpha)(y)= \begin{cases}\alpha(y) & \text { if } y \neq x \\ \sum_{i=1}^{k} \alpha\left(t a_{i}\right)-\alpha(x) & \text { otherwise. }\end{cases}
$$

Finally it is known (see [5] and [6]) that for every $x \in Q_{0}$ sink or a source of $Q$ we can define respectively the functors

$$
C_{x}^{+}: \operatorname{Rep}(Q) \rightarrow \operatorname{Rep}\left(c_{x}(Q)\right) \quad \text { and } \quad C_{x}^{-}: \operatorname{Rep}(Q) \rightarrow \operatorname{Rep}\left(c_{x}(Q)\right)
$$

called reflection functors.

Definition 1.1. Let $Q$ be a quiver with $n$ vertices without oriented cycles. We choose the numbering $\left(x_{1}, \ldots, x_{n}\right)$ of vertices such that ta $>$ ha for every $a \in Q_{1}$. We define

$$
C^{+}:=C_{x_{n}}^{+} \cdots C_{x_{1}}^{+} \quad \text { and } \quad C^{-}:=C_{x_{1}}^{-} \cdots C_{x_{n}}^{-} .
$$

The functors $C^{+}, C^{-}: \operatorname{Rep}(Q) \rightarrow \operatorname{Rep}(Q)$ are called Coxeter functors.

One proves that these functors don't depend on the choice of numbering of vertices (see [4] chap. VII Lemma 5.8).

Theorem 1.2 (Auslander-Reiten 1975). Let Q be a quiver without oriented cycles.

1) For every indecomposable non-projective representation $V$ of $Q$ there is an almost split sequence $0 \rightarrow$ $C^{+} V \rightarrow X \rightarrow V \rightarrow 0$ in $\operatorname{Rep}(Q)$.

2) For every indecomposable non-injective representation $V$ of $Q$ there is an almost split sequence $0 \rightarrow V \rightarrow$ $Z \rightarrow C^{-} V \rightarrow 0$ in $\operatorname{Rep}(Q)$. 
Proof. See [4], sec. IV.3 Theorem 3.1.

Definition 1.3. A quiver $Q$ is called of tame type if the underlying graph of $Q$ is of type $\widetilde{A}, \widetilde{D}$ or $\widetilde{E}$.

For all next results we refer to [6]. We also consider $C^{+}$and $C^{-}$as linear transformations on the space of dimension vectors, i.e. if $V$ is a representation of a quiver with dimension $\alpha$ then $C^{ \pm} \alpha=\underline{\operatorname{dim}}\left(C^{ \pm} V\right)$.

Proposition 1.4. Let $Q$ be a quiver of tame type, then the quadratic form $q_{Q}: \mathbb{Z}^{Q_{0}} \rightarrow \mathbb{Z}$ defined by

$$
q_{Q}(\alpha):=\sum_{x \in Q_{0}} \alpha(x)^{2}-\sum_{a \in Q_{1}} \alpha(t a) \alpha(h a)
$$

is positive semi-definite and there exists a unique vector $h \in \mathbb{N} Q_{0}$ such that $\mathbb{Z} h$ is the radical of $q_{Q}$. For quivers of type $\widetilde{A}$ and $\widetilde{D}$ the vector $h$ has the following form

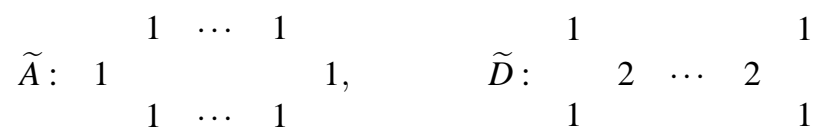

Definition 1.5. Let $V$ be an indecomposable representation of $Q$.

(i) $V$ is preprojective if and only if $\left(C^{+}\right)^{i} V=0$ for $i>>0$.

(ii) $V$ is preinjective if and only if $\left(C^{-}\right)^{i} V=0$ for $i>>0$.

(iii) $V$ is regular if and only if $\left(C^{+}\right)^{i} V \neq 0$ for every $i \in \mathbb{Z}$.

Definition 1.6. Let $V$ be a representation of $Q$. The linear map

$$
\partial: \mathbb{N}^{Q_{0}} \longrightarrow \mathbb{Z}
$$

defined by $\partial(\underline{\operatorname{dim}} V):=\langle h, \underline{\operatorname{dim}} V\rangle$ is called defect of $V$.

Lemma 1.7. Let $V$ an indecomposable representation of $Q . V$ is preprojective, preinjective or regular if and only if the defect of $V$ is respectively negative, positive or zero.

The regular representations of $Q$ form an Abelian category $\operatorname{Reg}(Q)$. Moreover $\operatorname{Reg}(Q)$ is serial, i.e. every indecomposable regular representation has only one regular composition series and so it is only determined by its regular socle and by its regular length.

Definition 1.8. A simple regular module $E$ is called homogeneous if and only if $\underline{\operatorname{dim}} E=h$.

Proposition 1.9. Let $Q$ be a quiver of tame type. Then there exist at most three $C^{+}$-orbits $\Delta=\left\{e_{i} \mid i \in\right.$ $I=\{0, \ldots, u\}\}, \Delta^{\prime}=\left\{e_{i}^{\prime} \mid i \in I^{\prime}=\{0, \ldots, v\}\right\}, \Delta^{\prime \prime}=\left\{e_{i}^{\prime \prime} \mid i \in I^{\prime \prime}=\{0, \ldots, w\}\right\}$, of dimension vectors of nonhomogeneous simple regular representations of $Q\left(I, I^{\prime}, I^{\prime \prime}\right.$ could be empty). We can assume that $C^{+}\left(e_{i}\right)=e_{i+1}$ for $i \in I\left(e_{u+1}=e_{0}\right), C^{+}\left(e_{i}^{\prime}\right)=e_{i+1}^{\prime}$ for $i \in I^{\prime}\left(e_{v+1}^{\prime}=e_{0}^{\prime}\right)$ and $C^{+}\left(e_{i}^{\prime \prime}\right)=e_{i+1}^{\prime \prime}$ for $i \in I^{\prime \prime}\left(e_{w+1}^{\prime \prime}=e_{0}\right)$.

Graphycally we can present $\Delta, \Delta^{\prime}$ and $\Delta^{\prime \prime}$ respectively as the polygons
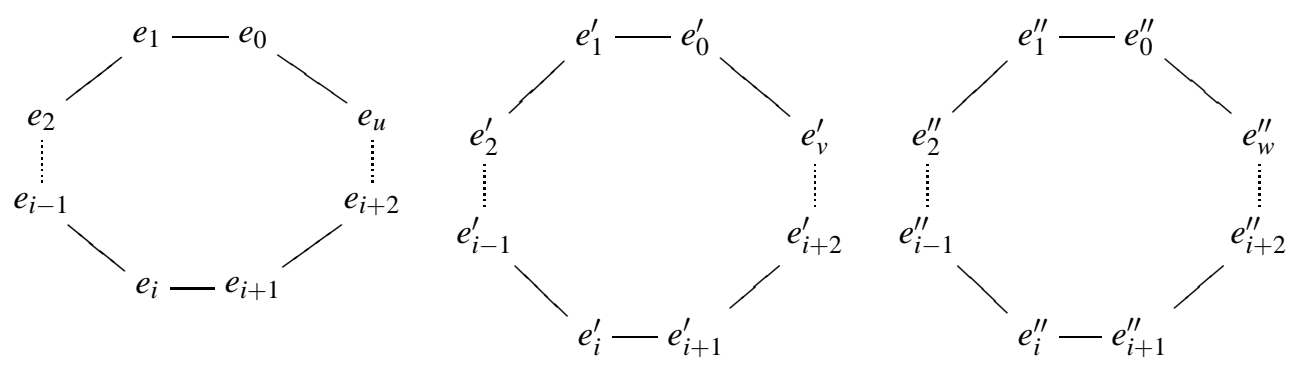
Every dimension vector $d$ of a regular representation of $Q$ can be decomposed uniquely as

$$
d=p h+\sum_{i \in I} p_{i} e_{i}+\sum_{i \in I^{\prime}} p_{i}^{\prime} e_{i}^{\prime}+\sum_{i \in I^{\prime \prime}} p_{i}^{\prime \prime} e_{i}^{\prime \prime}
$$

for some $p, p_{i}, p_{i}^{\prime}, p_{i}^{\prime \prime} \in \mathbb{N}$ such that at least one of coefficients in each family $\left\{p_{i} \mid i \in I\right\},\left\{p_{i}^{\prime} \mid i \in I^{\prime}\right\},\left\{p_{i}^{\prime \prime} \mid i \in I^{\prime \prime}\right\}$ is zero.

We observe that the category $\operatorname{Reg}(Q)$ can be decomposed as direct sum of categories $\mathscr{R}_{t}$, with $t=(\varphi, \psi) \in$ $\mathbb{P}_{1}(\mathbb{k})$. In all categories $\mathscr{R}_{t}$, but at most three of these, there is only one simple object $V_{t}$ which is necessarily homogeneous.

Definition 1.10. (1) We call $E_{i}, E_{i}^{\prime}$ and $E_{i}^{\prime \prime}$ the simple non-homogeneous regular representations respectively of dimension $e_{i}, e_{i}^{\prime}$ and $e_{i}^{\prime \prime}$.

(2) We call $V_{(\varphi, \psi)}$, where $(\varphi, \psi) \in \mathbb{P}_{1}(\mathbb{k})$, the indecomposable regular representation of dimension $h$.

(3) We define $E_{i, j}$ to be the indecomposable regular representations with socle $E_{i}$ and dimension $\sum_{k=i}^{j} e_{k}$, where $e_{k}$ are vertices of the arc with clockwise orientation $e_{i}-\cdots . . . .-e_{j}$ in $\Delta$, without repetitions of $e_{k}$. We denote $E_{i}:=E_{i, i}$ and similarly we define $E_{i, j}^{\prime}$ and $E_{i, j}^{\prime \prime}$.

To simplify the notation, sometimes we will consider, without advance notice, $I=\{1, \ldots, u+1\}$ (respectively $I^{\prime}=\{1, \ldots, v+1\}$ ), identifying $E_{i, u+1}$ with $E_{i, 0}$ and $E_{u+1, i}$ with $E_{0, i}$ for every $i \in I$ (respectively $E_{i, v+1}^{\prime}$ with $E_{i, 0}^{\prime}$ and $E_{v+1, i}^{\prime}$ with $E_{0, i}^{\prime}$ for every $\left.i \in I^{\prime}\right)$.

\subsection{Symmetric quivers}

Definition 1.11. A symmetric quiver is a pair $(Q, \sigma)$ where $Q$ is a quiver (called underlying quiver of $(Q, \sigma)$ ) and $\sigma$ is an involution on $Q_{0} \sqcup Q_{1}$ such that

(i) $\sigma\left(Q_{0}\right)=Q_{0}$ and $\sigma\left(Q_{1}\right)=Q_{1}$,

(ii) $t \sigma(a)=\sigma(h a)$ and $h \sigma(a)=\sigma(t a)$ for all $a \in Q_{1}$,

(iii) $\sigma(a)=a$ whenever $a \in Q_{1}$ and $\sigma(t a)=h a$.

Let $V$ be a representation of the underlying quiver $Q$ of a symmetric quiver $(Q, \sigma)$. We define the duality functor $\nabla: \operatorname{Rep}(Q) \rightarrow \operatorname{Rep}(Q)$ such that $\nabla V(x)=V(\sigma(x))^{*}$ for every $x \in Q_{0}$ and $\nabla V(a)=-V(\sigma(a))^{*}$ for every $a \in Q_{1}$. If $f: V \rightarrow W$ is a morphism of representations $V, W \in \operatorname{Rep}(Q)$, then $\nabla f: \nabla W \rightarrow \nabla V$ is defined by $\nabla f(x)=f(\sigma(x))^{*}$, for every $x \in Q_{0}$. We call $V$ selfdual if $\nabla V=V$.

Definition 1.12. An orthogonal (resp. symplectic) representation of a symmetric quiver $(Q, \sigma)$ is a pair $(V,<$ $\cdot, \cdot>)$, where $V$ is a representation of the underlying quiver $Q$ with a nondegenerate symmetric (resp. skewsymmetric) scalar product $<\cdot, \cdot>$ on $\bigoplus_{x \in Q_{0}} V(x)$ such that

(i) the restriction of $\langle\cdot, \cdot>$ to $V(x) \times V(y)$ is 0 if $y \neq \sigma(x)$,

(ii) $\langle V(a)(v), w>+<v, V(\sigma(a))(w)>=0$ for all $v \in V($ ta $)$ and all $w \in V(\sigma(a))$.

By properties $(i)$ and $(i i)$ of definition 1.12, an orthogonal or symplectic representation $(V,<\cdot, \cdot>)$ of a symmetric quiver is selfdual.

We shall say that a dimension vector $\alpha$ is symmetric if $\alpha(x)=\alpha(\sigma(x))$ for every $x \in Q_{0}$. Since each orthogonal or symplectic representation is selfdual, then dimension vector of an orthogonal (resp. symplectic) representation, which we shall call respectively orthogonal and symplectic dimension vector, is symmetric.

Definition 1.13. An orthogonal (respectively symplectic) representation is called indecomposable orthogonal (respectively indecomposable symplectic) if it cannot be expressed as a direct sum of orthogonal (respectively symplectic) representations. 
Definition 1.14. A symmetric quiver is said to be of tame representation type if is not of finite representation type, but in every dimension vector the indecomposable orthogonal (symplectic) representations occur in families of dimension $\leq 1$.

Derksen and Weyman classified the symmetric quiver of tame type in [8]

Proposition 1.15. Let $(Q, \sigma)$ be a symmetric tame quiver with $Q$ connected. Then $(Q, \sigma)$ is one of the following symmetric quivers.

(1) Of type
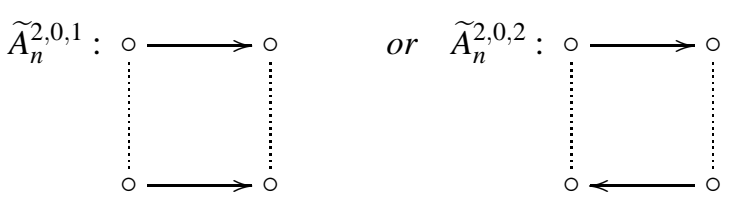

with arbitrary orientation reversed under $\sigma$ if $Q=\widetilde{A}_{2 n+1}(n \geq 1)$. Here $\sigma$ is a reflection with respect to a central vertical line (so $\sigma$ fixes two arrows and no vertices).

(2) Of type

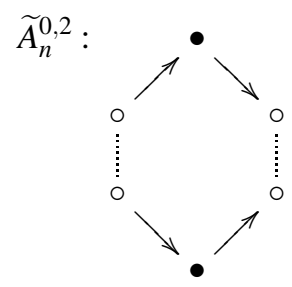

with arbitrary orientation reversed under $\sigma$ if $Q=\widetilde{A}_{2 n-1}(n \leq 1)$. Here $\sigma$ is a reflection with respect to a central vertical line (so $\sigma$ fixes two vertices and no arrows).

(3) Of type

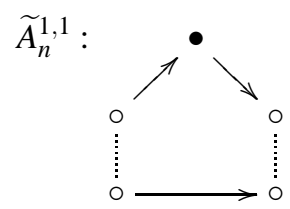

with arbitrary orientation reversed under $\sigma$ if $Q=\widetilde{A}_{2 n}(n \geq 1)$. Here $\sigma$ is a reflection with respect to a central vertical line (so $\sigma$ fixes one arrow and one vertex).

(4) Of type

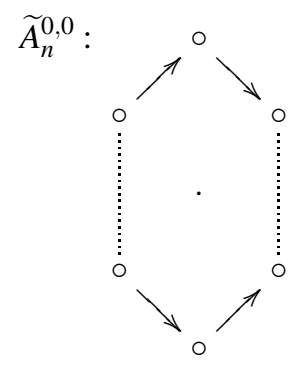

with arbitrary orientation reversed under $\sigma$ if $Q=\widetilde{A}_{2 n+1}(n \geq 1)$. Here $\sigma$ is a central symmetry (so $\sigma$ fixes neither arrows nor vertices). 
(5) Of type

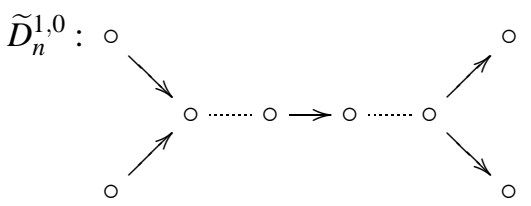

with arbitrary orientation reversed under $\sigma$ if $Q=\widetilde{D}_{2 n}(n \geq 2)$. Here $\sigma$ is a reflection with respect to a central vertical line (so $\sigma$ fixes one arrow and no vertices).

(6) Of type

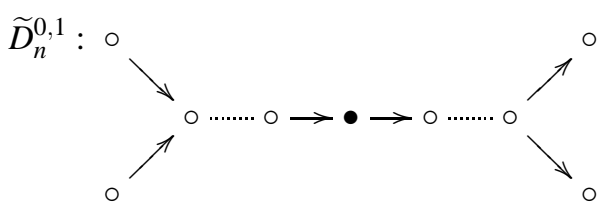

with arbitrary orientation reversed under $\sigma$ if $Q=\widetilde{D}_{2 n-1}(n \geq 2)$. Here $\sigma$ is a reflection with respect to a central vertical line (so $\sigma$ fixes one vertex and no arrows).

Proof. See [8], proposition 4.3.

We describe the space of orthogonal (resp. symplectic) representations of a symmetric quiver $(Q, \sigma)$.

We denote $Q_{0}^{\sigma}$ (respectively $Q_{1}^{\sigma}$ ) the set of vertices (respectively arrows) fixed by $\sigma$. Thus we have partitions

$$
\begin{aligned}
& Q_{0}=Q_{0}^{+} \sqcup Q_{0}^{\sigma} \sqcup Q_{0}^{-} \\
& Q_{1}=Q_{1}^{+} \sqcup Q_{1}^{\sigma} \sqcup Q_{1}^{-}
\end{aligned}
$$

such that $Q_{0}^{-}=\sigma\left(Q_{0}^{+}\right)$and $Q_{1}^{-}=\sigma\left(Q_{1}^{+}\right)$, satisfying:

i) $\forall a \in Q_{1}^{+}$, either $\{t a, h a\} \subset Q_{0}^{+}$or one of the elements in $\{t a, h a\}$ is in $Q_{0}^{+}$while the other is in $Q_{0}^{\sigma}$;

ii) $\forall x \in Q_{0}^{+}$, if $a \in Q_{1}$ with $t a=x$ or $h a=x$, then $a \in Q_{1}^{+} \sqcup Q_{1}^{\sigma}$.

Definition 1.16. Let $(Q, \sigma)$ be a symmetric quiver. We define a linear map $\delta: \mathbb{N} Q_{0} \rightarrow \mathbb{N} Q_{0}$ by setting $\{\delta \alpha(i)\}_{i \in Q_{0}}=$ $\{\alpha(\sigma(i))\}_{i \in Q_{0}}$ for every dimension vector $\alpha$.

Remark 1.17. (i) Since $\sigma$ is an involution, also $\delta$ is one.

(ii) If $V$ is a representation of dimension $\alpha$ then $\delta \alpha=\underline{\operatorname{dim}}(\nabla V)$. In particular if $V$ is an orthogonal or symplectic representation of $(Q, \sigma)$ of dimension $\alpha$, then $\delta \alpha=\alpha$.

(iii) If $\alpha$ and $\beta$ are dimension vectors, then

$$
\langle\alpha, \beta\rangle=\langle\delta \beta, \delta \alpha\rangle .
$$

For next statements, see section 2 in [8]. Let $\operatorname{ORep}(Q, \alpha)$ be the space of orthogonal $\alpha$-dimensional representations of a symmetric quiver $(Q, \sigma)$; by property (ii) of definition 1.12 we have

$$
\operatorname{ORep}(Q, \alpha) \cong \bigoplus_{a \in Q_{1}^{+}} \operatorname{Hom}\left(\mathbb{k}^{\alpha(t a)}, \mathbb{k}^{\alpha(h a)}\right) \oplus \bigoplus_{a \in Q_{1}^{\sigma}} \bigwedge^{2}\left(\mathbb{k}^{\alpha(t a)}\right)^{*}
$$

Let $\operatorname{SpRep}(Q, \alpha)$ be the space of symplectic $\alpha$-dimensional representations of a symmetric quiver $(Q, \sigma)$; by property (ii) of definition 1.12 we have

$$
\operatorname{SpRep}(Q, \alpha) \cong \bigoplus_{a \in Q_{1}^{+}} \operatorname{Hom}\left(\mathbb{k}^{\alpha(t a)}, \mathbb{k}^{\alpha(h a)}\right) \oplus \bigoplus_{a \in Q_{1}^{\sigma}} S_{2}\left(\mathbb{k}^{\alpha(t a)}\right)^{*}
$$


By property $(i)$ of definition 1.12 , the subgroup of $\operatorname{SL}(Q, \alpha)$ which stabilizes $\operatorname{ORe}(Q, \alpha)$ is

$$
S O(Q, \alpha)=\prod_{x \in Q_{0}^{+}} S L(\alpha(x)) \times \prod_{x \in Q_{0}^{\sigma}} S O(\alpha(x)),
$$

where $S O(\alpha(x))$ is the group of special orthogonal transformations for the symmetric form $\langle\cdot, \cdot\rangle$ restricted to $V(x)$.

Assuming that $\alpha(x)$ is even for every $x \in Q_{0}^{\sigma}$, by property $(i)$ of definition 1.12 , the subgroup of $S L(Q, \alpha)$ which stabilizes $\operatorname{SpRep}(Q, \alpha)$ is

$$
\operatorname{SSp}(Q, \alpha)=\prod_{x \in Q_{0}^{+}} \operatorname{SL}(\alpha(x)) \times \prod_{x \in Q_{0}^{\sigma}} \operatorname{Sp}(\alpha(x)),
$$

where $\operatorname{Sp}(\alpha(x))$ is the group of isometric transformations for the skew-symmetric form $\langle\cdot, \cdot\rangle$ restricted to $V(x)$.

The action of these groups is defined by

$$
g \cdot V=\left\{g_{h a} V(a) g_{t a}^{-1}\right\}_{a \in Q_{1}^{+} \cup Q_{1}^{\delta}}
$$

where $g=\left(g_{x}\right)_{x \in Q_{0}} \in \operatorname{SO}(Q, \alpha)$ (respectively $g \in \operatorname{SSp}(Q, \alpha)$ ) and $V \in \operatorname{ORep}(Q, \alpha)$ (respectively in $\operatorname{SpRep}(Q, \alpha)$ ). In particular we can suppose $g_{\sigma(x)}=\left(g_{x}^{-1}\right)^{t}$ for every $x \in Q_{0}$.

\subsection{Semi-invariants of quivers without oriented cycles and main results}

In this section first we define semi-invariants which appear in main results of paper and we describe some property of these for any quiver $Q$; then we state main theorems.

Let $Q$ be a quiver with $n$ vertices. We denote

$$
S I(Q, \alpha)=\mathbb{k}[\operatorname{Rep}(Q, \alpha)]^{S L(Q, \alpha)}
$$

the ring of semi-invariants of a quiver $Q$.

For every $g \in G L(Q, \alpha)$ the character $\tau$ at $g$ is $\tau(g)=\operatorname{det}(g)^{\chi_{1}} \cdots \operatorname{det}(g)^{\chi_{n}}$, where $\chi=\left(\chi_{1}, \ldots, \chi_{n}\right) \in \mathbb{Z}^{n}$ is also called weight if $\tau$ is a weight for some semi-invariant. So the ring $\operatorname{SI}(Q, \alpha)$ decomposes in graded components as

$$
S I(Q, \alpha)=\bigoplus_{\tau \in \operatorname{char}(G L(Q, \alpha))} \operatorname{SI}(Q, \alpha)_{\tau}
$$

where $S I(Q, \alpha)_{\tau}=\{f \in \mathbb{k}[\operatorname{Rep}(Q, \alpha)] \mid g \cdot f=\tau(g) f \forall g \in G L(Q, \alpha)\}$.

For every $V \in \operatorname{Rep}(Q, \alpha)$, we can construct a projective resolution, called canonical resolution of $V$ :

$$
0 \longrightarrow \bigoplus_{a \in Q_{1}} V(t a) \otimes P_{h a} \stackrel{d^{V}}{\longrightarrow} \bigoplus_{x \in Q_{0}} V(x) \otimes P_{x} \stackrel{p_{V}}{\longrightarrow} V \longrightarrow 0
$$

where $P_{x}$ is the indecomposable projective associated to vertex $x$ for every $x \in Q_{0}$ (see [18]), $\left.d^{V}\right|_{V(t a) \otimes P_{h a}}(v \otimes$ $\left.e_{h a}\right)=V(a)(v) \otimes e_{h a}-v \otimes a$ and $\left.p_{V}\right|_{V(x) \otimes P_{x}}(v)=v \otimes e_{x}$. Applying the functor $\operatorname{Hom}_{Q}(\cdot, W)$ to $d^{V}$ for $W \in$ $\operatorname{Rep}(Q, \beta)$, we have that the matrix associated to $\operatorname{Hom}_{Q}\left(d^{V}, W\right)$ is square if and only if $\langle\alpha, \beta\rangle=0$ (see [20] Lemma 1.2).

Definition 1.18. For $V \in \operatorname{Rep}(Q, \alpha)$ such that $\langle\alpha, \beta\rangle=0$, where $\beta \in \mathbb{N}^{n}$, we define

$$
\begin{aligned}
c^{V}: \operatorname{Rep}(Q, \beta) & \longrightarrow \mathbb{k} \\
W & \longmapsto c^{V}(W)=\operatorname{det}\left(\operatorname{Hom}_{Q}\left(d^{V}, W\right)\right) .
\end{aligned}
$$

These are semi-invariants of weight $\langle\alpha, \cdot\rangle$, called Schofield semi-invariants (see [20] Lemma 1.4). 
Remark 1.19. (i) If

$$
0 \rightarrow P_{1} \stackrel{\varphi}{\rightarrow} P_{0} \rightarrow V \rightarrow 0
$$

is another projective resolution of $V$ and $\langle\alpha, \beta\rangle=0$, than

$$
\operatorname{det}\left(\operatorname{Hom}_{Q}(\varphi, \cdot)\right)=k \cdot \operatorname{det}\left(\operatorname{Hom}_{Q}\left(d^{V}, \cdot\right)\right)
$$

for some $k \in \mathbb{k}$ (see [20] lemma 1.2). So any projective resolution of $V$ can be used to calculate $c^{V}$ (see $[20]$ ). Moreover if $P$ is a projective representation, then $c^{P}=0$.

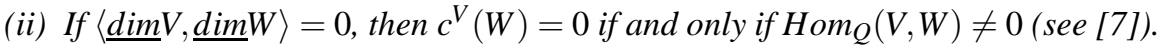

Now we formulate the result of Derksen and Weyman about the set of generators of the ring of semiinvariants of a quiver without oriented cycles $Q$.

Theorem 1.20 (Derksen-Weyman). Let $Q$ be a quiver without oriented cycles and let $\beta$ be a dimension vector. The ring $\operatorname{SI}(Q, \beta)$ is spanned by semi-invariants of the form $c^{V}$ of weight $\langle\underline{\operatorname{dim}}(V), \cdot\rangle$, for which $\langle\underline{\operatorname{dim}}(V), \beta\rangle=0$.

Proof. See [7] Theorem 1.

We give some property of Schofield semi-invariants.

Lemma 1.21. Suppose that $V^{\prime}, V, V^{\prime \prime}$ and $W$ are representations of $Q$, that $\langle\underline{\operatorname{dim}}(V), \underline{\operatorname{dim}}(W)\rangle=0$ and that there are exact sequences

$$
0 \rightarrow V^{\prime} \rightarrow V \rightarrow V^{\prime \prime} \rightarrow 0
$$

then

(i) If $\left\langle\underline{\operatorname{dim}}\left(V^{\prime}\right), \underline{\operatorname{dim}}(W)\right\rangle<0$, then $c^{V}(W)=0$

(ii) If $\left\langle\underline{\operatorname{dim}}\left(V^{\prime}\right), \underline{\operatorname{dim}}(W)\right\rangle=0$, then $c^{V}(W)=c^{V^{\prime}}(W) c^{V^{\prime \prime}}(W)$.

Proof. See [7] Lemma 1.

We recall definition and properties of the Pfaffian of a skew-symmetric matrix.

Let $A=\left(a_{i j}\right)_{1 \leq i, j \leq 2 n}$ be a skew-symmetric $2 n \times 2 n$ matrix. Given $2 n$ vectors $x_{1}, \ldots, x_{2 n}$ in $\mathbb{k}^{2 n}$, we define

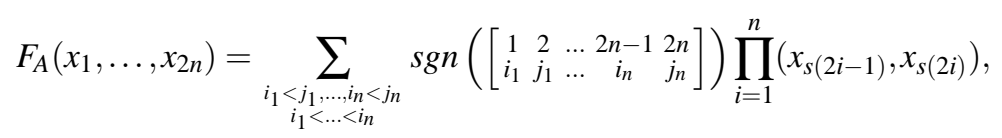

where $\operatorname{sgn}(\cdot)$ is the sign of the permutation and $(\cdot, \cdot)$ is the skew-symmetric bilinear form associated to $A$. So $F_{A}$ is a skew-symmetric multilinear function of $x_{1}, \ldots, x_{2 n}$. Since, up to a scalar, the only one skew-symmetric multilinear function of $2 n$ vectors in $\mathbb{k}^{2 n}$ is the determinant, there is a complex number $p f(A)$, called Pfaffian of $A$, such that

$$
F_{A}\left(x_{1}, \ldots, x_{2 n}\right)=p f(A) \operatorname{det}\left[x_{1}, \ldots, x_{2 n}\right]
$$

where $\left[x_{1}, \ldots, x_{2 n}\right]$ is the matrix which has the vector $x_{i}$ for $i$-th column. In particular we note that

$$
p f(A)=\sum_{\substack{i_{1}<j_{1}, \ldots, i_{n}<j_{n} \\
i_{1}<\ldots<i_{n}}} \operatorname{sgn}\left(\left[\begin{array}{ccccc}
1 & 2 & \ldots & 2 n-1 & 2 n \\
i_{1} & j_{1} & \ldots & i_{n} & j_{n}
\end{array}\right]\right) a_{1_{1} j_{1}} \cdots a_{i_{n} j_{n}} .
$$

Proposition 1.22. Let $A$ be a skew-symmetric $2 n \times 2 n$ matrix.

(i) For every invertible $2 n \times 2 n$ matrix $B$,

$$
p f\left(B A B^{t}\right)=\operatorname{det}(B) p f(A) ;
$$


(ii) $\operatorname{det}(A)=p f(A)^{2}$.

Proof. See [17], chap. 5 sec. 3.6.

Let

$$
0 \longrightarrow P_{1} \stackrel{d_{\min }^{V}}{\longrightarrow} P_{0} \longrightarrow V \longrightarrow 0
$$

be the minimal projective resolution of $V \in \operatorname{Rep}(Q, \alpha)$ and let $\beta$ be a dimension vector such that $\langle\alpha, \beta\rangle=0$, we will prove (Lemma 3.1) that, under some hypothesis, $\operatorname{Hom}_{Q}\left(d_{\text {min }}^{V}, \cdot\right)$ is skew-symmetric on $\operatorname{Rep}(Q, \beta)$, so we can define

$$
\begin{aligned}
p f^{V}: \operatorname{Rep}(Q, \beta) & \longrightarrow \mathbb{k} \\
W & \longmapsto p f^{V}(W)=\operatorname{Pf}\left(\operatorname{Hom}_{Q}\left(d_{\text {min }}^{V}, W\right)\right) .
\end{aligned}
$$

In this work we describe a set of generators of the rings of semi-invariants of symmetric quivers of tame type. Let $\alpha$ be a dimension vector of an orthogonal or symplectic representation, we denote

$$
\operatorname{OSI}(Q, \alpha):=\mathbb{k}[\operatorname{ORep}(Q, \alpha)]^{\operatorname{SO}(Q, \alpha)} \quad \text { and } \operatorname{SpSI}(Q, \alpha):=\mathbb{k}[\operatorname{SpRep}(Q, \alpha)]^{\operatorname{SSp}(Q, \alpha)}
$$

respectively the ring of orthogonal semi-invariants and the ring of symplectic semi-invariants of a symmetric quiver $(Q, \sigma)$.

We state the main theorems

Theorem 1.23. Let $(Q, \sigma)$ be a symmetric quiver of tame type and let $\beta$ be a regular symmetric dimension vector. The ring $\operatorname{SpSI}(Q, \beta)$ is generated by semi-invariants

(i) $c^{V}$ if $V \in \operatorname{Rep}(Q)$ is such that $\langle\underline{\operatorname{dim}} V, \beta\rangle=0$,

(ii) $p f^{V}$ if $V \in \operatorname{Rep}(Q)$ is such that $\langle\underline{\operatorname{dim} V}, \beta\rangle=0, C^{+} V=\nabla V$ and the almost split sequence $0 \rightarrow \nabla V \rightarrow Z \rightarrow$ $V \rightarrow 0$ has the middle term $Z$ in $\operatorname{ORep}(Q)$.

Theorem 1.24. Let $(Q, \sigma)$ be a symmetric quiver of tame type let $\beta$ be a regular symmetric dimension vector. The ring $\operatorname{OSI}(Q, \beta)$ is generated by semi-invariants

(i) $c^{V}$ if $V \in \operatorname{Rep}(Q)$ is such that $\langle\underline{\operatorname{dim} V}, \beta\rangle=0$,

(ii) $p f^{V}$ if $V \in \operatorname{Rep}(Q)$ is such that $\langle\underline{\operatorname{dim}} V, \beta\rangle=0, C^{+} V=\nabla V$ and the almost split sequence $0 \rightarrow \nabla V \rightarrow Z \rightarrow$ $V \rightarrow 0$ has the middle term $Z$ in $\operatorname{SpRep}(Q)$.

In section 3.1, we prove theorems 1.23 and 1.24 for $\beta=p h$; in section 3.2, for any regular symmetric dimension vector $\beta$.

Definition 1.25. Let $(Q, \sigma)$ be a symmetric quiver. We will say that $V \in \operatorname{Rep}(Q)$ satisfies property $(O p)$ if

(i) $V=C^{-} \nabla V$

(ii) the almost split sequence $0 \rightarrow \nabla V \rightarrow Z \rightarrow V \rightarrow 0$ has the middle term $Z$ in ORep $(Q)$.

Similarly we will say that $V \in \operatorname{Rep}(Q)$ satisfies property (Spp) if

(i) $V=C^{-} \nabla V$

(ii) the almost split sequence $0 \rightarrow \nabla V \rightarrow Z \rightarrow V \rightarrow 0$ has the middle term $Z$ in $\operatorname{SpRep}(Q)$. 


\subsection{Invariant theory and Schur modules}

Let $G$ be an algebraic group, $V$ a rational representation of $G$ and $\mathbb{k}[V]$ the algebra of regular functions of $V$. If $\mathscr{X}(G)$ is the set of characters of $G$, then the ring of the semi-invariants of $G$ on $V$ is defined by

$$
S I(G, V)=\bigoplus_{\chi \in \mathscr{X}(G)} S I(G, V)_{\chi}
$$

where $\operatorname{SI}(G, V)_{\chi}=\{f \in \mathbb{k}[V] \mid g \cdot f=\chi(g) f, \forall g \in G\}$ is called weight space of weight $\chi$. The following lemma describes $S I(G, V)$ in the case when $G$ has an open orbit on $V$.

Lemma 1.26 (Sato-Kimura). Let $G$ be a connected linear algebraic group and $V$ a rational representation of $G$. We suppose that the action of $G$ on $V$ has an open orbit. Then $\operatorname{SI}(G, V)$ is a polynomial $\mathbb{k}$-algebra and the weights of the generators of $\operatorname{SI}(G, V)$ are linearly independent in $\mathscr{X}(G)$. Moreover, the dimensions of the spaces $\operatorname{SI}(G, V)_{\chi}$ are 0 or 1 .

Proof. See [23], sect. 4, Lemma 4 and Proposition 5.

Let $G=G L_{n}(\mathbb{k})$ be the general linear group over $\mathbb{k}$. There exists an isomorphism $\mathbb{Z} \cong \mathscr{X}(G)$ which sends an element $a$ of $\mathbb{Z}$ in $(\text { det })^{a}$ (where det associates to $g \in G$ its determinant). We identify $G$ with the group $G L(V)$ of linear automorphisms of a vector space $V$ of dimension $n$. So we have

$$
S I(G, V)=\mathbb{k}[V]^{S L(V)} .
$$

Let $T$ and $\mathscr{X}(T)$ respectively be the maximal torus in $G$ (i.e. the group of diagonal matrices) and the set of characters of $T$. The irreducible rational representations of $G$ are parametrized by the set

$$
\mathscr{X}^{+}(T)=\left\{\lambda=\left(\lambda_{1}, \ldots, \lambda_{n}\right) \in \mathbb{Z}^{n} \mid \lambda_{1} \geq \cdots \geq \lambda_{n}\right\}
$$

of the integral dominant weights for $G L_{n}(\mathbb{k})$. The irreducible rational representations $S_{\lambda} V$ of $G=G L_{n}(\mathbb{k})$ corresponding to the dominant weight $\lambda \in \mathscr{X}^{+}(T)$ are called Schur modules. In the case when $\lambda_{n} \geq 0$ (i.e. $\lambda$ is a partition of $\lambda_{1}+\cdots+\lambda_{n}$ ), a description of $S_{\lambda} V$ is given in [1] and [17]. For every $\lambda \in \mathscr{X}^{+}(T)$, we can define $S_{\lambda} V$ as follows

$$
S_{\left(\lambda_{1}, \ldots, \lambda_{n}\right)} V=S_{\left(\lambda_{1}-\lambda_{n}, \ldots, \lambda_{n-1}-\lambda_{n}, 0\right)} V \otimes\left(\bigwedge^{n} V\right)^{\otimes \lambda_{n}} .
$$

Let $\lambda=\left(\lambda_{1}, \ldots, \lambda_{n}\right)$ be a partition of $|\lambda|:=\lambda_{1}+\cdots+\lambda_{n}$. We call height of $\lambda$, denoted by $h t(\lambda)$, the number $k$ of nonzero components of $\lambda$ and we denote the transpose of $\lambda$ by $\lambda^{\prime}$.

Theorem 1.27 (Properties of Schur modules). Let $V$ be vector space of dimension $n$ and $\lambda$ be an integral dominant weight.

(i) $S_{\lambda} V=0 \Leftrightarrow h t(\lambda)>0$.

(ii) $\operatorname{dim} S_{\lambda} V=1 \Leftrightarrow \lambda=(\overbrace{k, \ldots, k}^{n})$ for some $n \in \mathbb{Z}$.

(iii) $\left(S_{\left(\lambda_{1}, \ldots, \lambda_{n}\right)} V\right)^{*} \cong S_{\left(\lambda_{1}, \ldots, \lambda_{n}\right)} V^{*} \cong S_{\left(-\lambda_{n}, \ldots,-\lambda_{1}\right)} V$.

Proof. See Theorem 6.3 in [10].

Theorem 1.28 (Cauchy formulas). Let $V$ and $W$ be two finite dimensional vector spaces. Then

(i) As representations of $G L(V) \times G L(W)$,

$$
S_{d}(V \otimes W)=\bigoplus_{|\lambda|=d} S_{\lambda} V \otimes S_{\lambda} W \quad \text { and } \quad \bigwedge^{d}(V \otimes W)=\bigoplus_{|\lambda|=d} S_{\lambda} V \otimes S_{\lambda^{\prime}} W .
$$


(ii) As representations of $G L(V)$,

$$
S_{d}\left(S_{2}(V)\right)=\bigoplus_{|\lambda|=d} S_{2 \lambda} V \quad \text { and } \quad S_{d}\left(\bigwedge^{2}(V)\right)=\bigoplus_{|\lambda|=d} S_{2 \lambda^{\prime}} V
$$

where $2 \lambda=\left(2 \lambda_{1}, \ldots, 2 \lambda_{k}\right)$ if $\lambda=\left(\lambda_{1}, \ldots, \lambda_{k}\right)$.

Proof. See [17] chap. 9 sec. 6.3 and sec 8.4, chap 11 sec. 4.5 .

The decomposition of tensor product of Schur modules is

$$
S_{\lambda} V \otimes S_{\mu} V=\bigoplus_{v} c_{\lambda \mu}^{v} S_{v} V
$$

where the coefficients $c_{\lambda \mu}^{v}$ are called Littlewood-Richardson coefficients.

There exists a combinatorial formula to calculate $c_{\lambda \mu}^{v}$, called Littlewood-Richardson rule (see in [17] chap. 12 sec. 5.3).

Finally we state other two results on Schur modules and invariant theory.

Proposition 1.29. Let $V$ be a vector space of dimension $n$.

$$
\left(S_{\lambda} V\right)^{S L(V)} \neq 0 \quad \Longleftrightarrow \lambda=\left(k^{n}\right)
$$

for some $k$ and in this case $S_{\lambda} V$, and so also $\left(S_{\lambda} V\right)^{S L(V)}$, have dimension one.

Proof. See Corollary p. 388 in [17].

Proposition 1.30. Let $V$ be a vector space of dimension $n$ and let $\lambda$ and $\mu$ be two integral dominant weights. Then

$$
S_{\lambda} V \otimes S_{\mu} V \neq 0 \quad \Longleftrightarrow \quad \lambda_{i}-\lambda_{i+1}=\mu_{n-i}-\mu_{n-i+1}
$$

for every $i \in\{1, \ldots, n-1\}$ and in this case the semi-invariant is unique (up to a non zero scalar) and has weight $\lambda_{1}+\mu_{n}=\lambda_{2}+\mu_{n-1}=\cdots=\lambda_{n}+\mu_{1}$.

Proof. It is a Corollary of (7.11) in [15] chap. 1 sec. 5.

Proposition 1.31. Let $V$ be an orthogonal space of dimension $n$ and let $W$ be a symplectic space of dimension $2 n$.

$$
\operatorname{dim}\left(S_{\lambda} V\right)^{S O(V)}=\left\{\begin{array}{ll}
1 & \text { if } \lambda=2 \mu+\left(k^{n}\right) \\
0 & \text { otherwise }
\end{array} \quad \text { and } \quad \operatorname{dim}\left(S_{\lambda} W\right)^{S p(W)}= \begin{cases}1 & \text { if } \lambda=2 \mu^{\prime} \\
0 & \text { otherwise }\end{cases}\right.
$$

for some partition $\mu$ and for some $k \in \mathbb{N}$.

Proof. See [17] chap. 11 corollaries 5.2.1 and 5.2.2.

\section{Reflection functors and semi-invariants of symmetric quivers}

\subsection{Reflection functors for symmetric quivers}

We adjust the technique of reflection functors to symmetric quivers. See [3] section 2.1 for the proofs of results of this paragraph.

Definition 2.1. Let $(Q, \sigma)$ be a symmetric quiver. A sink (resp. a source) $x \in Q_{0}$ is called admissible if there are no arrows connecting $x$ and $\sigma(x)$.

By definition of $\sigma, x$ is an admissible sink (resp. a source) if and only if $\sigma(x)$ is an admissible source (resp. a sink). We call $(x, \sigma(x))$ the admissible sink-source pair. So we can define $c_{(x, \sigma(x))}:=c_{\sigma(x)} c_{x}$. Moreover we can prove that $\left(c_{(x, \sigma(x))} Q, \sigma\right)$ is a symmetric quiver (see Lemma 2.2 in [3]). 
Definition 2.2. Let $(Q, \sigma)$ be a symmetric quiver. A sequence $x_{1}, \ldots, x_{m} \in Q_{0}$ is an admissible sequence of sinks (or sources) for admissible sink-source pairs if $x_{i+1}$ is an admissible sink (resp. source) in $c_{\left(x_{i}, \sigma\left(x_{i}\right)\right)}{ }^{\cdots} c_{\left(x_{1}, \sigma\left(x_{1}\right)\right)}(Q)$ for $i=1, \ldots, m-1$.

The underlying graph of $\widetilde{D}$ is a tree, so by Proposition 2.4 in [3], applying a composition of reflections at admissible sink-source pairs, from any orientation of $\widetilde{D}$ we can get the following orientation

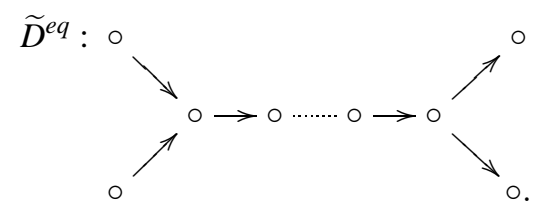

Definition 2.3. We will say that a symmetric quiver is of type $(s, t, k, l)$ if

(i) it is of type $\widetilde{A}$,

(ii) $\left|Q_{1}^{\sigma}\right|=s$ and $\left|Q_{0}^{\sigma}\right|=t$,

(iii) it has $k$ counterclockwise arrows and l clockwise arrows in $Q_{1}^{+} \sqcup Q_{1}^{-}$.

By Proposition 1.15, $s, t \in\{0,1,2\}$ and if either $s$ or $t$ are not zero, then $s+t=2$. Moreover, by symmetry, we note that $k$ and $l$ have to be even.

One proves, restricting to subquivers of type $A$, by a simple combinatorial argument the following (for further details, see Proposition 1.3.8 in [2])

Proposition 2.4. Let $(Q, \sigma)$ be a symmetric quiver of type $\widetilde{A}$ such that $Q$ is without oriented cycles. Then there is an admissible sequence of sinks $x_{1}, \ldots, x_{s}$ of $Q$ for admissible sink-source pairs such that $c_{\left(x_{1}, \sigma\left(x_{1}\right)\right)} \cdots c_{\left(x_{s}, \sigma\left(x_{s}\right)\right)} Q$ is one of the quivers:

(1)

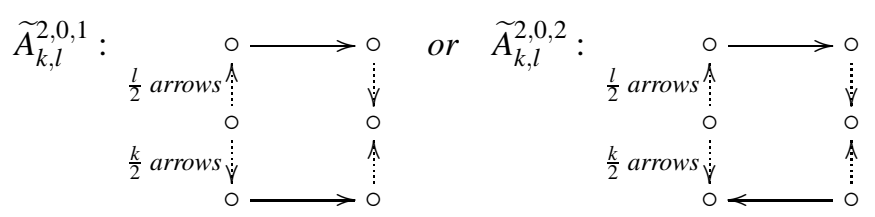

if $(Q, \sigma)$ is of type $(2,0, k, l)$;

(2)

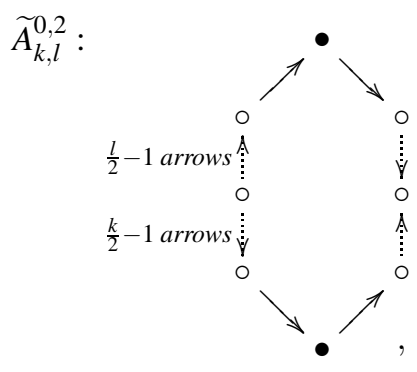

if $(Q, \sigma)$ is of type $(0,2, k, l)$;

(3)

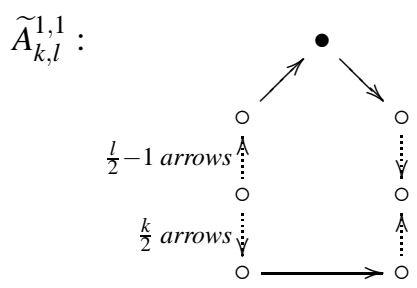

if $(Q, \sigma)$ is of type $(1,1, k, l)$; 
(4)

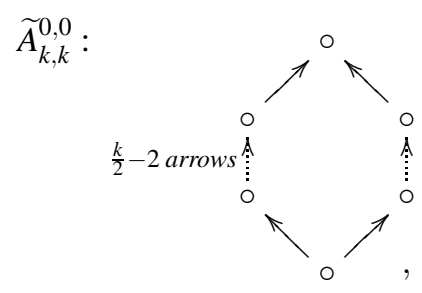

if $(Q, \sigma)$ if of type $(0,0, k, k)$.

Let $(Q, \sigma)$ be a symmetric quiver and $(x, \sigma(x))$ a sink-source admissible pair. For every $V \in \operatorname{Rep}(Q)$, we define the reflection functors

$$
C_{(x, \sigma(x))}^{+} V:=C_{\sigma(x)}^{-} C_{x}^{+} V \quad \text { and } \quad C_{(x, \sigma(x))}^{-} V:=C_{x}^{-} C_{\sigma(x)}^{+} V .
$$

Proposition 2.5. Let $(Q, \sigma)$ and $\left(Q^{\prime}, \sigma\right)$ be two symmetric quivers with the same underlying graph. We suppose that $Q^{\prime}=c_{\left(x_{m}, \sigma\left(x_{m}\right)\right)} \cdots c_{\left(x_{1}, \sigma\left(x_{1}\right)\right)}(Q)$ for some admissible sequence of sinks $x_{1}, \ldots, x_{m} \in Q_{0}$ for admissible sinksource pairs and let $V^{\prime}=C_{\left(x_{m}, \sigma\left(x_{m}\right)\right)}^{+} \cdots C_{\left(x_{1}, \sigma\left(x_{1}\right)\right)}^{+} V \in \operatorname{Rep}\left(Q^{\prime}\right)$. Then

$$
V=C^{-} \nabla V \Leftrightarrow V^{\prime}=C^{-} \nabla V^{\prime} .
$$

Proof. See Corollary 2.6 in [3].

Proposition 2.6. Let $(Q, \sigma)$ be a symmetric quiver and let $x$ be an admissible sink. Then $V$ is an orthogonal (resp. symplectic) representation of $(Q, \sigma)$ if and only if $C_{(x, \sigma(x))}^{+} V$ is an orthogonal (resp. symplectic) representation of $\left(c_{(x, \sigma(x))} Q, \sigma\right)$. Similarly for $C_{(x, \sigma(x))}^{-}$if $x$ is an admissible source.

Proof. See Proposition 2.7 in [3].

\subsection{Orthogonal and symplectic semi-invariants}

If $W$ is a vector space of dimension $n$, we denote $\widetilde{G r}(r, W)$ the set of all decomposable tensors $w_{1} \wedge \ldots \wedge w_{r}$, with $w_{1}, \ldots, w_{r} \in W$, inside $\bigwedge^{r} W$.

Lemma 2.7. If $x$ is an admissible sink or source for a symmetric quiver $(Q, \sigma)$ and $\alpha$ is a dimension vector such that $c_{(x, \sigma(x))} \alpha(x) \geq 0$, then

i) if $c_{(x, \sigma(x))} \alpha(x)>0$, then there exist isomorphisms

$$
\operatorname{SpSI}(Q, \alpha) \stackrel{\varphi_{x, \alpha}^{S p}}{\longrightarrow} \operatorname{SpSI}\left(c_{(x, \sigma(x))} Q, c_{(x, \sigma(x))} \alpha\right) \quad \text { and } \quad \operatorname{OSI}(Q, \alpha) \stackrel{\varphi_{x, \alpha}^{O}}{\longrightarrow} \operatorname{OSI}\left(c_{(x, \sigma(x))} Q, c_{(x, \sigma(x))} \alpha\right) ;
$$

ii) if $c_{(x, \sigma(x))} \alpha(x)=0$, then there exist isomorphisms

$$
\operatorname{SpSI}(Q, \alpha) \stackrel{\varphi_{x, \alpha}^{S p}}{\longrightarrow} \operatorname{SpSI}\left(c_{(x, \sigma(x))} Q, c_{(x, \sigma(x))} \alpha\right)[y] \quad \text { and } \quad \operatorname{OSI}(Q, \alpha) \stackrel{\varphi_{x, \alpha}^{O}}{\longrightarrow} \operatorname{OSI}\left(c_{(x, \sigma(x))} Q, c_{(x, \sigma(x))} \alpha\right)[y]
$$

where $R[y]$ denotes a polynomial ring with coefficients in $R$.

Proof. See [3], Lemma 2.8

In next section we will see that for tame type $\varphi_{x, \alpha}^{S p}$ and $\varphi_{x, \alpha}^{O}$ send $c^{V}$ to $c^{C_{(x, \sigma(x))}^{+} V}$ (propositions 3.3 and 3.4). We recall that, by definition, symplectic groups or orthogonal groups act on the spaces which are defined on the vertices in $Q_{0}^{\sigma}$, so we have 
Definition 2.8. Let $V$ be a representation of the underlying quiver $Q$ with $\underline{\operatorname{dim} V}=\alpha$ such that $\langle\alpha, \beta\rangle=0$ for some symmetric dimension vector $\beta$. The weight of $c^{V}$ on $\operatorname{SpRep}(Q, \beta)$ (respectively on $\left.\operatorname{ORep}(Q, \beta)\right)$ is $\langle\alpha, \cdot\rangle-\sum_{x \in Q_{0}^{\sigma}} \varepsilon_{x, \alpha}$, where

$$
\varepsilon_{x, \alpha}(y)= \begin{cases}\langle\alpha, \cdot\rangle(x) & y=x \\ 0 & \text { otherwise. }\end{cases}
$$

We shall say that a weight is symmetric if $\chi(i)=-\chi(\sigma(i))$ for every $i \in Q_{0}$.

Remark 2.9. Let $(Q, \sigma)$ be a symmetric quiver and $V \in \operatorname{Rep}(Q, \alpha)$. We note that

$$
\left\langle\underline{\operatorname{dim}}\left(C^{-} \nabla V\right), \cdot\right\rangle(i)=-\langle\alpha, \cdot\rangle(\sigma(i))
$$

for every $i \in Q_{0}$. So, if $C^{-} \nabla V=V$ then $\chi=\langle\alpha, \cdot\rangle$ is a symmetric weight.

Lemma 2.10. Let $(Q, \sigma)$ be a symmetric quiver. For every representation $V$ of the underlying quiver $Q$ and for every orthogonal or symplectic representation $W$ such that $\langle\underline{\operatorname{dim}}(V), \underline{\operatorname{dim}}(W)\rangle=0$, we have

$$
c^{V}(W)=c^{C^{-} \nabla V}(W) .
$$

Proof. See [3], Corollary 2.16.

We conclude this section with two lemma which will be useful later.

Lemma 2.11. Let

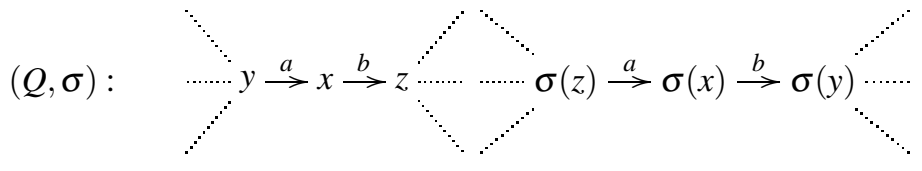

be a symmetric quiver. Assume there exist only two arrows in $Q_{1}^{+}$incident to $x \in Q_{0}^{+}, a: y \rightarrow x$ and $b: x \rightarrow z$ with $y, z \in Q_{0}^{+} \cup Q_{0}^{\sigma}$. Let $V$ be an orthogonal or symplectic representation with symmetric dimension vector $\left(\alpha_{i}\right)_{i \in Q_{0}}=\alpha$ such that $\alpha_{x} \geq \max \left\{\alpha_{y}, \alpha_{z}\right\}$.

We define the symmetric quiver $Q^{\prime}=\left(\left(Q_{0}^{\prime}, Q_{1}^{\prime}\right), \sigma\right)$ with $n-2$ vertices such that $Q_{0}^{\prime}=Q_{0} \backslash\{x, \sigma(x)\}$ and $Q_{1}^{\prime}=$ $Q_{1} \backslash\{a, b, \sigma(a), \sigma(b)\} \cup\{b a, \sigma(a) \sigma(b)\}$ and let $\alpha^{\prime}$ be the dimension of $V$ restricted to $Q^{\prime}$.

We have:

(Sp) Assume V symplectic. Then

(a) if $\alpha_{x}>\max \left\{\alpha_{y}, \alpha_{z}\right\}$ then $\operatorname{SpSI}(Q, \alpha)=\operatorname{SpSI}\left(Q^{\prime}, \alpha^{\prime}\right)$,

(b) if $\alpha_{x}=\alpha_{y}>\alpha_{z}$ then $\operatorname{SpSI}(Q, \alpha)=\operatorname{SpSI}\left(Q^{\prime}, \alpha^{\prime}\right)[\operatorname{det} V(a)]$,

(b') if $\alpha_{x}=\alpha_{z}>\alpha_{y}$ then $\operatorname{SpSI}(Q, \alpha)=\operatorname{SpSI}\left(Q^{\prime}, \alpha^{\prime}\right)[\operatorname{det} V(b)]$,

(c) if $\alpha_{x}=\alpha_{y}=\alpha_{z}$ then $\operatorname{SpSI}(Q, \alpha)=\operatorname{SpSI}\left(Q^{\prime}, \alpha^{\prime}\right)[\operatorname{det} V(a), \operatorname{det} V(b)]$.

(O) Assume V orthogonal. Then

(a) if $\alpha_{x}>\max \left\{\alpha_{y}, \alpha_{z}\right\}$ then $\operatorname{OSI}(Q, \alpha)=\operatorname{OSI}\left(Q^{\prime}, \alpha^{\prime}\right)$,

(b) if $\alpha_{x}=\alpha_{y}>\alpha_{z}$ then $\operatorname{OSI}(Q, \alpha)=\operatorname{OSI}\left(Q^{\prime}, \alpha^{\prime}\right)[\operatorname{det} V(a)]$,

(b') if $\alpha_{x}=\alpha_{z}>\alpha_{y}$ then $\operatorname{OSI}(Q, \alpha)=\operatorname{OSI}\left(Q^{\prime}, \alpha^{\prime}\right)[\operatorname{det} V(b)]$,

(c) if $\alpha_{x}=\alpha_{y}=\alpha_{z}$ then $\operatorname{OSI}(Q, \alpha)=\operatorname{OSI}\left(Q^{\prime}, \alpha^{\prime}\right)[\operatorname{det} V(a), \operatorname{det} V(b)]$.

Proof. See [3], Lemma 2.17.

Similarly one proves the following 
Lemma 2.12. Let

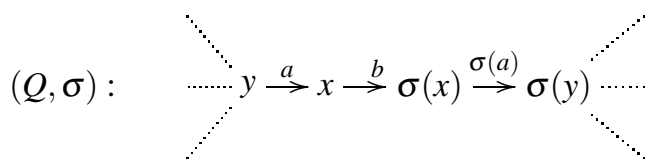

be a symmetric quiver with $n$ vertices such that there exist only two arrows a and $b$ incident to the vertex $x$ in $Q_{0}$ and $b$ is fixed by $\sigma$. Let $V$ be an orthogonal or symplectic representation of $(Q, \sigma)$ with $\underline{\operatorname{dim}}(V)=\alpha$ such that $\alpha_{x} \geq \alpha_{y}$. Moreover we define the symmetric quiver $\left(Q^{\prime}, \sigma\right)=\left(\left(Q_{0}^{\prime}, Q_{1}^{\prime}\right), \sigma\right)$ with $n-2$ vertices such that $Q_{0}^{\prime}=Q_{0} \backslash\{x, \sigma(x)\}$ and $Q_{1}^{\prime}=Q_{1} \backslash\{a, b, \sigma(a)\} \cup\{\sigma(a) b a\}$.

Let $\alpha^{\prime}$ be the dimension of $V$ restricted to $Q^{\prime}$.

(Sp) If $V$ is symplectic, then

(i) $\alpha_{x}>\alpha_{y} \Longrightarrow \operatorname{SpSI}(Q, \alpha)=\operatorname{SpSI}\left(Q^{\prime}, \alpha^{\prime}\right)[\operatorname{det} V(b)]$

(ii) $\alpha_{x}=\alpha_{y} \Longrightarrow \operatorname{SpSI}(Q, \alpha)=\operatorname{SpSI}\left(Q^{\prime}, \alpha^{\prime}\right)[\operatorname{det} V(a)]$.

(O) If $V$ is orthogonal, then

(i) $\alpha_{x}>\alpha_{y}$ and $\alpha_{x}$ is even $\Longrightarrow \operatorname{OSI}(Q, \alpha)=\operatorname{OSI}\left(Q^{\prime}, \alpha^{\prime}\right)[p f V(b)]$

(ii) $\alpha_{x}=\alpha_{y} \Longrightarrow \operatorname{OSI}(Q, \alpha)=\operatorname{OSI}\left(Q^{\prime}, \alpha^{\prime}\right)[\operatorname{det} V(a)]$.

\section{Semi-invariants of symmetric quivers of tame type}

In this section we prove theorems 1.23 and 1.24 for the symmetric quivers of tame type. We recall that the underlying quiver of a symmetric quiver of tame type is either $\widetilde{A}$ or $\widetilde{D}$ as in Proposition 1.15 . As done for the finite case in [3], we again reduce the proof to particular orientations (orientations in Proposition 2.4 for $\widetilde{A}$ and orientation of $\widetilde{D}^{e q}$ for $\widetilde{D}$ ).

We start with some result which will be useful later.

Lemma 3.1. Let $(Q, \sigma)$ be a symmetric quiver of tame type. Let $d_{\min }^{V}$ be the matrix of the minimal projective presentation of $V \in \operatorname{Rep}(Q, \alpha)$ and let $\beta$ be a symmetric dimension vector such that $\langle\alpha, \beta\rangle=0$. Then

(1) $\operatorname{Hom}_{Q}\left(d_{\text {min }}^{V}, \cdot\right)$ is skew-symmetric on $\operatorname{SpRep}(Q, \beta)$ if and only if $V$ satisfies property $(O p)$;

(2) $\operatorname{Hom}_{Q}\left(d_{\text {min }}^{V}, \cdot\right)$ is skew-symmetric on $\operatorname{ORep}(Q, \beta)$ if and only if $V$ satisfies property (Spp).

Proof. First we note, by Auslander-Reiten quiver of $Q$, that if $(Q, \sigma)$ is a symmetric quiver of tame type, then the only representations $V \in \operatorname{Rep}(Q)$ such that $C^{-} \nabla V=V$ are regular ones.

We prove (1) only for symmetric quivers $(Q, \sigma)$ of type $(1,1, k, l)$ (see Definition 2.3), because for (2) and the other cases one proceeds similarly (for the details for symmetric quiver of type $\widetilde{D}_{n}^{0,1}$, see Lemma 1.4.6 in [2]). We call $\left(Q^{\prime}, \sigma\right)$ the symmetric quiver with the same underlying graph of $(Q, \sigma)$ and with orientation as in Proposition 2.4 By Proposition 2.4 there exists a sequence $x_{1}, \ldots, x_{m}$ of admissible sink for admissible sinksource pairs such that $c_{\left(x_{m}, \sigma\left(x_{m}\right)\right)} \cdots c_{\left(x_{1}, \sigma\left(x_{1}\right)\right)} Q=Q^{\prime}$. We call $V^{\prime}:=C_{\left(x_{m}, \sigma\left(x_{m}\right)\right)}^{+} \cdots C_{\left(x_{1}, \sigma\left(x_{1}\right)\right)}^{+} V$ for every $V \in$ $\operatorname{Rep}(Q)$ and if $\alpha=\underline{\operatorname{dim}} V$, then $\alpha^{\prime}:=c_{\left(x_{m}, \sigma\left(x_{m}\right)\right)} \cdots c_{\left(x_{1}, \sigma\left(x_{1}\right)\right)} \alpha$. We note that, by Proposition 2.5 and Proposition 2.6. $V$ satisfies property $(O p)$ (respectively property $(S p p)$ ) if and only if $V^{\prime}$ satisfies property $(O p)$ (respectively property $(S p p))$. 
We consider the following labelling for $Q^{\prime}=\widetilde{A}_{k, l}^{1,1}$ :

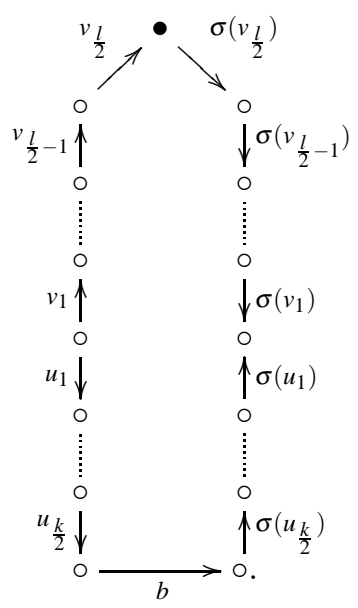

Let $E_{i, j}^{h}$ be the regular indecomposable representation of dimension $e_{i, j}+h$ which contains $E_{i, j}$ (similarly we define $E_{i, j}^{\prime}$ ). From the regular component of the Auslander-Reiten quiver of $Q^{\prime}$, we note that the following indecomposable representations $V^{\prime} \in \operatorname{Rep}\left(Q^{\prime}\right)$ satisfy property $(O p)$ (the other regular indecomposable representations of $\operatorname{Rep}\left(Q^{\prime}\right)$ satisfying property $(O p)$ are extensions of these).

(a) $V_{(0,1)}$ such that $Z^{\prime}=E_{1}^{h} \oplus E_{0,2}=\nabla Z^{\prime}$.

(b) $E_{i-1, j}$, with $1 \leq j<i-1 \leq l$, such that $\nabla E_{i-1, j}=E_{i, j+1}$ and $Z^{\prime}=E_{i+1, j-1} \oplus E_{i, j}=\nabla Z^{\prime}$.

(c) $E_{i-1, j}^{h}$, with $1 \leq i-1<j \leq l$, such that $\nabla E_{i-1, j}^{h}=E_{i, j+1}^{h}$ and $Z^{\prime}=E_{i+1, j-1}^{h} \oplus E_{i, j}^{h}=\nabla Z^{\prime}$.

(d) $E_{i-1, j}^{\prime}$, with $1 \leq i-1<j \leq k+1$, such that $\nabla E_{i-1, j}^{\prime}=E_{i, j+1}^{\prime}$ and $Z^{\prime}=E_{i+1, j-1}^{\prime} \oplus E_{i, j}^{\prime}=\nabla Z^{\prime}$.

(e) $E_{i-1, j}^{\prime h}$, with $1 \leq j \leq i-1 \leq k+1$, such that $\nabla E_{i-1, j}^{\prime h}=E_{i, j+1}^{\prime h}$ and $Z^{\prime}=E_{i+1, j-1}^{\prime h} \oplus E_{i, j}^{\prime h}=\nabla Z^{\prime}$.

If $V$ is the middle term of a short exact sequence $0 \rightarrow V^{1} \rightarrow V \rightarrow V^{2} \rightarrow 0$, with $V^{1}$ and $V^{2}$ one of the representations of type (a), (b), (c), (d) or (e), we have the blocks matrix

$$
\operatorname{Hom}_{Q}\left(d_{\text {min }}^{V}, \cdot\right)=\left(\begin{array}{cc}
\operatorname{Hom}_{Q}\left(d_{\text {min }}^{V^{1}}, \cdot\right) & 0 \\
\operatorname{Hom}_{Q}(B, \cdot) & \operatorname{Hom}_{Q}\left(d_{\text {min }}^{V^{2}}, \cdot\right)
\end{array}\right) .
$$

where $d_{\text {min }}^{V^{1}}: P_{1}^{1} \rightarrow P_{0}^{1}$ is the minimal projective presentation of $V^{1}, d_{\text {min }}^{V^{2}}: P_{1}^{2} \rightarrow P_{0}^{2}$ is the minimal projective presentation of $V^{2}$ and for some $B \in \operatorname{Hom}_{Q}\left(P_{1}^{2}, P_{0}^{1}\right)$. In general for every blocks matrix we have $\left(\begin{array}{cc}A & 0 \\ 0 & C\end{array}\right)=$ $\left(\begin{array}{cc}I d & 0 \\ -B A^{-1} & I d\end{array}\right) \cdot\left(\begin{array}{cc}A & 0 \\ B & C\end{array}\right)$ if $A$ is invertible. Hence using rows operations on $\operatorname{Hom}_{Q}\left(d_{\text {min }}^{V}, \cdot\right)$, we obtain

$$
\operatorname{Hom}_{Q}\left(d_{\text {min }}^{V}, \cdot\right) \approx\left(\begin{array}{cc}
\operatorname{Hom}_{Q}\left(d_{\text {min }}^{V^{1}}, \cdot\right) & 0 \\
0 & \operatorname{Hom}_{Q}\left(d_{\text {min }}^{V^{2}}, \cdot\right)
\end{array}\right) .
$$

So it's enough to prove the skew-symmetry of $\operatorname{Hom}_{Q}\left(d_{\min }^{V}, \cdot\right)$ on $\operatorname{SpRep}(Q, \beta)$ for $V$ one of representations of type (a), (b), (c), (d) and (e).

Let $\chi$ be the symmetric weight associated to $\alpha$. We order vertices of $Q$ clockwise from $t b=1$ to $h b=k+l+1$. Let $W \in \operatorname{SpRep}(Q, \beta)$. We prove that $\operatorname{Hom}_{Q}\left(d_{\text {min }}^{V}, W\right)$ is skew-symmetric for every regular indecomposable representation $V$ of type (a), (b), (c), (d) and (e). First we observe that the associated to $V$ symmetric weight 
$\chi$ have components equal to 0,1 and -1 . Let $m_{1}$ be the first vertex such that $\chi\left(m_{1}\right) \neq 0$ and $m_{s}$ the last vertex such that $\chi\left(m_{s}\right) \neq 0$. Between -1 and 1 alternate in correspondence respectively of sinks and of sources. In particular, $\chi\left(m_{1}\right)= \pm 1=-\chi\left(m_{s}\right)$ and $\chi\left(m_{i}\right)=1$ or -1 , for every $i \in\{2, \ldots, s-1\}$, respectively if $m_{i}$ is a source or a sink. We note that, for every $\operatorname{Hom}_{Q}\left(d_{\text {min }}^{V}, W\right)$ with $V$ one representation of type (a), (b), (c), (d) and (e), we can restrict to the symmetric subquiver of type $A$ which has first vertex $m_{1}$ and last vertex $m_{s}$ and passing through the $\sigma$-fixed vertex of $Q$. Hence it proceeds as done for type $A$ (see Lemma 3.1 in [3]).

Definition 3.2. For $V \in \operatorname{Rep}(Q, \alpha)$ satisfying property (Spp) (resp. satisfying property (Op) such that $\langle\alpha, \beta\rangle=$ 0 , where $\beta$ is an orthogonal (resp. symplectic) dimension vector, we define

$$
\begin{aligned}
p f^{V}: \operatorname{Rep}(Q, \beta) & \longrightarrow \mathbb{k} \\
W & \longmapsto c^{V}(W)=p f\left(\operatorname{Hom}_{Q}\left(d_{\text {min }}^{V}, W\right)\right) .
\end{aligned}
$$

Proposition 3.3. Let $(Q, \sigma)$ be a symmetric quiver of tame type. Let $\alpha$ be a symmetric dimension vector, $x$ be an admissible sink and $\varphi_{x, \alpha}^{S p}$ be as defined in Lemma 2.7

Then $\varphi_{x, \alpha}^{S p}\left(c^{V}\right)=c^{C_{(x, \sigma(x))}^{+} V}$ and $\varphi_{x, \alpha}^{S p}\left(p f^{W}\right)=p f_{(x, \sigma(x))}^{C^{+}}$, where $V$ and $W$ are indecomposables of $Q$ such that $\langle\underline{\operatorname{dim}} V, \alpha\rangle=0=\langle\underline{\operatorname{dim}} W, \alpha\rangle$ and $W$ satisfies property $(O p)$. In particular

(i) if $0=\alpha_{x} \neq \sum_{a \in Q_{1}: t a=x} \alpha_{t a}$, then $\left(\varphi_{x, \alpha}^{S p}\right)^{-1}\left(c^{S_{x}}\right)=0$;

(ii) if $0 \neq \alpha_{x}=\sum_{a \in Q_{1}: t a=x} \alpha_{t a}$, then $\varphi_{x, \alpha}^{S p}\left(c^{S_{\sigma(x)}}\right)=0$.

Proof. See Proposition 3.3 in [3].

Proposition 3.4. Let $(Q, \sigma)$ be a symmetric quiver of tame type. Let $\alpha$ be a symmetric dimension vector, $x$ be an admissible sink and $\varphi_{x, \alpha}^{O}$ be as defined in Lemma 2.7

Then $\varphi_{x, \alpha}^{O}\left(c^{V}\right)=c^{C_{(x, \sigma(x))}^{+} V}$ and $\varphi_{x, \alpha}^{O}\left(p f^{W}\right)=p f^{C_{(x, \sigma(x))}^{+}}$, where $V$ and $W$ are indecomposables of $Q$ such that $\langle\underline{\operatorname{dim}} V, \alpha\rangle=0=\langle\underline{\operatorname{dim}} W, \alpha\rangle$ and $W$ satisfies property (Spp). In particular

(i) if $0=\alpha_{x} \neq \sum_{a \in Q_{1}: t a=x} \alpha_{t a}$, then $\left(\varphi_{x, \alpha}^{O}\right)^{-1}\left(c^{S_{x}}\right)=0$;

(ii) if $0 \neq \alpha_{x}=\sum_{a \in Q_{1}: t a=x} \alpha_{t a}$, then $\varphi_{x, \alpha}^{O}\left(c^{S_{\sigma(x)}}\right)=0$.

Proof. See Proposition 3.4 in [3].

By Proposition 3.3. Proposition 3.4 and by Lemma 2.7, it follows that if Theorem 1.23 and Theorem 1.24 are true for $(Q, \sigma)$, then they are true for $\left(c_{(x, \sigma(x))} Q, \sigma\right)$.

\subsection{Semi-invariants of Symmetric quivers of tame type for dimension vector $p h$}

In this section we deal with dimension vector $p h$ (for definition of $h$, see Proposition 1.4). One proves Theorems 1.23 and 1.24 type by type of quivers $\widetilde{A}$ and $\widetilde{D}$. As said above, we can consider orientations of symmetric quivers of type $\widetilde{A}$ in Proposition 2.4 and orientation of symmetric quiver $\widetilde{D}^{e q}$. We proves these theorems only for $\widetilde{A}_{k, l}^{1,1}$. For the other type the proof is similar (for further details see sec 3.1 in [2]).

We shall call $\Lambda, E R \Lambda$ and $E C \Lambda$ respectively the set of partitions, the set of partition with even rows and the set of partition of even columns.

Theorems 1.23 and 1.24 for type $(1,1, k, l)$ follow from next theorem.

Theorem 3.5. Let $(Q, \sigma)$ be a symmetric quiver of type $(1,1, k, l)$ with orientation as in (11). Then O) $\operatorname{OSI}(Q, p h)$ is generated by the following indecomposable semi-invariants: if $p$ is even,

a) $\operatorname{det} V\left(u_{j}\right)$ with $j \in\left\{1, \ldots, \frac{k}{2}\right\}$;

b) $\operatorname{det} V\left(v_{j}\right)$ with $j \in\left\{1, \ldots, \frac{l}{2}\right\}$; 
c) $p f V(b)$

d) the coefficients $c_{i}$ of $\varphi^{p-2 i} \psi^{2 i}, 0 \leq i \leq \frac{p}{2}$, in $\operatorname{det}(\psi V(\sigma(\bar{a}) \bar{a})+\varphi V(\bar{b}))$, where $\bar{a}=v_{\frac{l}{2}} \cdots v_{1}$ and $\bar{b}=$ $\sigma\left(u_{1}\right) \cdots \sigma\left(u_{\frac{k}{2}}\right) b u_{\frac{k}{2}} \cdots u_{1}$;

if $p$ is odd,

a) $\operatorname{det} V\left(u_{j}\right)$ with $j \in\left\{1, \ldots, \frac{k}{2}\right\}$;

b) $\operatorname{det} V\left(v_{j}\right)$ with $j \in\left\{1, \ldots, \frac{l}{2}\right\}$;

c) the coefficients $c_{i}$ of $\varphi^{p-2 i} \psi^{2 i}, 0 \leq i \leq \frac{p-1}{2}$, in $\operatorname{det}(\psi V(\sigma(\bar{a}) \bar{a})+\varphi V(\bar{b}))$, where $\bar{a}=v_{\frac{l}{2}} \cdots v_{1}$ and $\bar{b}=$ $\sigma\left(u_{1}\right) \cdots \sigma\left(u_{\frac{k}{2}}\right) b u_{\frac{k}{2}} \cdots u_{1}$.

Sp) $\operatorname{SpSI}(Q, p h)$ is generated by the following indecomposable semi-invariants:

if $p$ is even,

a) $\operatorname{det} V\left(u_{j}\right)$ with $j \in\left\{1, \ldots, \frac{k}{2}\right\}$;

b) $\operatorname{det} V\left(v_{j}\right)$ with $j \in\left\{1, \ldots, \frac{l}{2}\right\}$;

c) $\operatorname{det} V(b)$

d) the coefficients $c_{i}$ of $\varphi^{p-2 i} \psi^{2 i}, 0 \leq i \leq \frac{p}{2}$, in $\operatorname{det}(\psi V(\sigma(\bar{a}) \bar{a})+\varphi V(\bar{b}))$, where $\bar{a}=v_{\frac{l}{2}} \cdots v_{1}$ and $\bar{b}=$ $\sigma\left(u_{1}\right) \cdots \sigma\left(u_{\frac{k}{2}}\right) b u_{\frac{k}{2}} \cdots u_{1}$;

if $p$ is odd, $\operatorname{SpSI}(Q, p h)=\mathbb{k}$.

Proof. We proceed by induction on $\frac{k}{2}+\frac{l}{2}$. The smallest case is $\widetilde{A}_{0,2}^{1,1}$

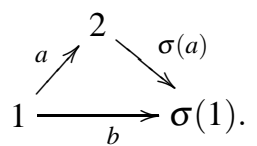

The induction step follows by Lemma 2.11 and by Lemma 2.12, so it's enough to prove the theorem for $\widetilde{A}_{0,2}^{1,1}$.

O) The ring of orthogonal semi-invariants is

$$
\bigoplus_{\substack{\lambda(a) \in \Lambda \\ \lambda(b) \in E C \Lambda}}\left(S_{\lambda(a)} V_{1} \otimes S_{\lambda(b)} V_{1}\right)^{S L V_{1}} \otimes\left(S_{\lambda(a)} V_{2}\right)^{S O V_{2}}
$$

By Proposition 1.30 we have

$$
\lambda(a)_{j}+\lambda(b)_{p-j+1}=k_{1}
$$

for every $0 \leq j \leq p$ and for some $k_{1} \in \mathbb{N}$. By Proposition 1.31 we have $\lambda(a)=2 \mu+\left(l^{p}\right)$ for some $\mu \in \Lambda$ and for some $l \in \mathbb{N}$. We consider the summands in which $k_{1}=1,2$ because the other ones are generated by products of powers of the generators of this summands.

Let $p$ be even. If $k_{1}=1$ the only solutions of (12) are $\lambda(a)=\left(1^{p}\right), \lambda(b)=0$ and $\lambda(a)=0, \lambda(b)=\left(1^{p}\right)$. Respectively, the summand $\left(S_{\left(1^{p}\right)} V_{1}\right)^{S L V_{1}} \otimes\left(S_{\left(1^{p}\right)} V_{2}\right)^{S O V_{2}}$ is generated by a semi-invariant of weight $(1,0)$, i.e $\operatorname{det} V(a)=\operatorname{det} V(\sigma(a))$, and the summand $\left(S_{\left(1^{p}\right)} V_{1}\right)^{S L V_{1}}$ is generated by a semi-invariant of weight $(1,0)$, i.e $p f V(b)$. If $k_{1}=2$, the solutions of (12) are $\lambda(a)=\left(2^{2 i}\right), \lambda(b)=\left(2^{p-2 i}\right)$ with $0 \leq i \leq \frac{p}{2}$. So the summand is

$$
\bigoplus_{i=0}^{\frac{p}{2}}\left(S_{\left(2^{2 i}\right)} V_{1} \otimes S_{\left(2^{p-2 i}\right)} V_{1}\right)^{S L V_{1}} \otimes\left(S_{\left(2^{2 i}\right)} V_{2}\right)^{S O V_{2}}
$$


which is generated by the coefficients of $\varphi^{p-2 i} \psi^{2 i}$ in $\operatorname{det}(\psi V(\sigma(a) a)+\varphi V(b))$, semi-invariants of weight $(2,0)$. In particular for $i=0$ we have $\operatorname{det} V(b)$ and for $i=\frac{p}{2}$ we have $\operatorname{det} V(\sigma(a) a)$.

Let $p$ be odd. If $k_{1}=1$ the only solutions of (12) are $\lambda(a)=\left(1^{p}\right), \lambda(b)=0$. The summand $\left(S_{\left(1^{p}\right)} V_{1}\right)^{S L V_{1}} \otimes$ $\left(S_{\left(1^{p}\right)} V_{2}\right)^{S O V_{2}}$ is generated by a semi-invariant of weight $(1,0)$, i.e $\operatorname{det} V(a)=\operatorname{det} V(\sigma(a))$. If $k_{1}=2$, the solutions of (12) are $\lambda(b)=\left(2^{2 i}\right), \lambda(a)=\left(2^{p-2 i}\right)$ with $0 \leq i \leq \frac{p-1}{2}$. So the summand is

$$
\bigoplus_{i=0}^{\frac{p-1}{2}}\left(S_{\left(2^{p-2 i}\right)} V_{1} \otimes S_{\left(2^{2 i}\right)} V_{1}\right)^{S L V_{1}} \otimes\left(S_{\left(2^{p-2 i}\right)} V_{2}\right)^{S O V_{2}}
$$

which is generated by the coefficients of $\varphi^{2 i} \psi^{p-2 i}$ in $\operatorname{det}(\psi V(\sigma(a) a)+\varphi V(b))$, semi-invariants of weight $(2,0)$.

Sp) The ring of symplectic semi-invariants is

$$
\bigoplus_{\substack{\lambda(a) \in \Lambda \\ \lambda(b) \in E R \Lambda}}\left(S_{\lambda(a)} V_{1} \otimes S_{\lambda(b)} V_{1}\right)^{S L V_{1}} \otimes\left(S_{\lambda(a)} V_{2}\right)^{S p V_{2}} .
$$

By Proposition 1.30 we have

$$
\lambda(a)_{j}+\lambda(b)_{p-j+1}=k_{1}
$$

for every $0 \leq j \leq p$ and for some $k_{1} \in \mathbb{N}$. By Proposition 1.31 we have $\lambda(a) \in E C \Lambda$. We consider the summands in which $k_{1}=1,2$ because the other ones are generated by products of powers of the generators of this summands.

Let $p$ be even. If $k_{1}=1$ the only solutions of $(13)$ are $\lambda(a)=\left(1^{p}\right), \lambda(b)=0$. The summand $\left(S_{\left(1^{p}\right)} V_{1}\right)^{S L V_{1}} \otimes$ $\left(S_{\left(1^{p}\right)} V_{2}\right)^{S p V_{2}}$ is generated by a semi-invariant of weight $(1,0)$, i.e $\operatorname{det} V(a)=\operatorname{det} V(\sigma(a))=\operatorname{pf} V(\sigma(a) a)$. If $k_{1}=2$, the solutions of (13) are $\lambda(a)=\left(2^{2 i}\right), \lambda(b)=\left(2^{p-2 i}\right)$ with $0 \leq i \leq \frac{p}{2}$. So the summand is

$$
\bigoplus_{i=0}^{\frac{p}{2}}\left(S_{\left(2^{2 i}\right)} V_{1} \otimes S_{\left(2^{p-2 i}\right)} V_{1}\right)^{S L V_{1}} \otimes\left(S_{\left(2^{2 i}\right)} V_{2}\right)^{S p V_{2}}
$$

which is generated by the coefficients of $\varphi^{p-2 i} \psi^{2 i}$ in $\operatorname{det}(\psi V(\sigma(a) a)+\varphi V(b))$, semi-invariants of weight $(2,0)$. In particular for $i=0$ we have $\operatorname{det} V(b)$ and for $i=\frac{p}{2}$ we have $\operatorname{det} V(\sigma(a) a)$.

If $p$ is odd there not exist any non-trivial symplectic representations because a symplectic space of dimension odd doesn't exist. So we have $\operatorname{SpSI}(Q, p h)=\mathbb{k}$.

From previous theorem, theorems 1.23 and 1.24 follow for symmetric quivers of type $(1,1, k, l)$.

Proof of Theorems 1.23 and 1.24 for type $(1,1, k, l)$. First of all we note that, by definition of $c^{W}$ and $p f^{W}$, when we have it, are not zero if $0=\langle\underline{\operatorname{dim}} W, p h\rangle=p\langle\underline{\operatorname{dim}} W, h\rangle=-p\langle h, \underline{\operatorname{dim}} W\rangle$, so we have to consider only regular representations $W$. Moreover it is enough to consider only simple regular representations $W$, because the other regular representations are extensions of simple regular ones and so, by Lemma 1.21, we obtain the $c^{W}$ and $p f^{W}$ with non-simple regular $W$ as products of those with simple regular $W$. Now we check only for $\widetilde{A}_{k, l}^{1,1}$ that the generators found, in previous theorem, for $\operatorname{SpSI}(Q, p h)$ and $\operatorname{SSI}(Q, p h)$ are of type $c^{W}$, for some simple regular $W$, and $p f^{W}$, for some simple regular $W$ satisfying property $(O p)$ in symplectic case and (Spp) in orthogonal case (see Lemma 3.1).

Sp) Let $V$ be a symplectic representation. We recall that, in this case, $p$ has to be even. The minimal projective resolution of $E_{0}$ is

$$
0 \longrightarrow P_{h\left(\sigma\left(v_{1}\right)\right)} \stackrel{d_{m i n}^{E_{0}}}{\longrightarrow} P_{t\left(\sigma\left(v_{1}\right)\right)} \longrightarrow E_{0} \longrightarrow 0
$$

where $d_{\min }^{E_{0}}=\sigma\left(v_{1}\right)$. So we have $c^{E_{0}}(V)=\operatorname{det}\left(V\left(\sigma\left(v_{1}\right)\right)\right)=\operatorname{det}\left(V\left(v_{1}\right)\right)=c^{E_{1}}(V)$. Similarly, we obtain $c^{E_{i}}(V)=\operatorname{det}\left(V\left(v_{i}\right)\right)=\operatorname{det}\left(V\left(\sigma\left(v_{i}\right)\right)\right)=c^{E_{l-i+1}}(V)$ for every $i \in\{2, \ldots, l\}, c^{E_{0}^{\prime}}(V)=\operatorname{det}\left(V\left(\sigma\left(u_{1}\right)\right)\right)=$ $\operatorname{det}\left(V\left(u_{1}\right)\right)=c^{E_{1}^{\prime}}(V), c^{E_{i}^{\prime}}(V)=\operatorname{det}\left(V\left(u_{i}\right)\right)=\operatorname{det}\left(V\left(\sigma\left(u_{i}\right)\right)\right)=c^{E_{k-i+2}^{\prime}(V)}$ for every $i \in\{2, \ldots, k\} \backslash\left\{\frac{k}{2}+\right.$ $1\}, c^{E_{k}^{\prime}+1}(V)=\operatorname{det}(V(b))$ and $c^{V_{(\varphi, \psi)}}(V)=\operatorname{det}(\psi V(\sigma(\bar{a}) \bar{a})+\varphi V(\bar{b}))$; 
O) if $V$ is an orthogonal representation, the only difference with the symplectic case is, when $p$ is even, we have $p f^{E_{\frac{k}{2}+1}^{\prime}}(V)=p f(V(b))$ and $p f^{V_{(\varphi, \psi)}}(V)=p f(\psi V(a)+\varphi V(b))$, in fact $E_{\frac{k}{2}+1}^{\prime}$ satisfies property $($ Spp $)$, since $C^{+} E_{\frac{k}{2}+1}^{\prime}=E_{\frac{k}{2}+2}^{\prime}=E_{\sigma\left(\frac{k}{2}+1\right)}^{\prime}=\nabla E_{\frac{k}{2}+1}^{\prime}$ and the almost split sequence

$$
0 \rightarrow E_{\frac{k}{2}+2}^{\prime} \rightarrow Z \rightarrow E_{\frac{k}{2}+1}^{\prime} \rightarrow 0
$$

has $Z=E_{\frac{k}{2}+2, \frac{k}{2}+1}^{\prime} \in \operatorname{SpRep}(Q)$.

\subsection{Semi-invariants of symmetric quivers of tame type for any regular dimension vec- tor}

In this section we prove theorems 1.23 and 1.24 for symmetric quivers of tame type and any regular symmetric dimension vector $d$.

We will use the same notation of section 3.1. For the type $\widetilde{A}$ we call $a_{0}=t v_{1}=t u_{1}, x_{i}=h v_{i}$ for every $i \in$ $\left\{1, \ldots, \frac{l}{2}\right\}$ and $y_{i}=h v_{i}$ for every $i \in\left\{1, \ldots, \frac{k}{2}\right\}$. For the type $\widetilde{D}$ we call $t_{1}=t a, t_{2}=t b$ and $z_{i}=t c_{i}$ for every $i$ such that $c_{i} \in\left(Q_{1}^{+} \sqcup Q_{1}^{\sigma}\right) \backslash\{a, b\}$.

First we consider the decomposition of $d$ for the symmetric quivers.

Let $(Q, \sigma)$ be a symmetric quiver of tame type and let $\Delta=\left\{e_{i} \mid i \in I=\{0, \ldots, u\}\right\}, \Delta^{\prime}=\left\{e_{i}^{\prime} \mid i \in I^{\prime}=\{0, \ldots, v\}\right\}$ and $\Delta^{\prime \prime}=\left\{e_{i}^{\prime \prime} \mid i \in I^{\prime \prime}=\{0, \ldots, w\}\right\}$ be the three $C^{+}$-orbits of nonhomogeneous simple regular representations of the underlying quiver $Q$ (see Proposition 1.9 ).

We shall call $I_{\delta}=\left\{i \in I \mid e_{i}=\delta e_{i}\right\}$ (respectively $I_{\delta}^{\prime}$ and $I_{\delta}^{\prime \prime}$ ). Let $[x]:=\max \{z \in \mathbb{N} \mid z \leq x\}$ be the floor of $x \in \mathbb{R}$.

Lemma 3.6. (a) For symmetric quivers of tame type with central symmetry, we have $\Delta=\Delta^{\prime}$ and so $I=I^{\prime}$.

(b) For symmetric quivers of tame type with symmetry respect to a central vertical line, we have decomposition $I=I_{+} \sqcup I_{\delta} \sqcup I_{-}$such that

(i) $I_{+}=\left\{2, \ldots,\left[\frac{u}{2}\right]+2\right\}, I_{\delta}=, I_{-}=I \backslash I_{+}$;

(ii) $I_{+}=\left\{2, \ldots,\left[\frac{u}{2}\right]+1\right\}, I_{\delta}=\{1\}, I_{-}=I \backslash\left(I_{+} \sqcup I_{\delta}\right)$;

(iii) $I_{+}=\left\{2, \ldots,\left[\frac{u}{2}\right]+1\right\}, I_{\delta}=\left\{1,\left[\frac{u}{2}\right]+2\right\}, I_{-}=I \backslash\left(I_{+} \sqcup I_{\delta}\right)$.

Similarly for $I^{\prime}$ but replacing $v$ with $u . I^{\prime \prime}=I_{+}^{\prime \prime} \sqcup I_{-}^{\prime \prime}$ such that $I_{+}^{\prime \prime}=\{2\}$ and $I_{-}^{\prime \prime}=I^{\prime \prime} \backslash I_{+}^{\prime \prime}$.

Proof. It follows by section 6 pages 40 and 46 in [6] and by definition of $\delta$ (Definition 1.16).

Proposition 3.7. Let $(Q, \sigma)$ be a symmetric quiver of tame type and let $I_{+}, I_{\delta}, I_{+}^{\prime}, I_{\delta}^{\prime}$ and $I_{+}^{\prime \prime}$ be as above. Any regular symmetric dimension vector can be written uniquely in the following form:

$$
d=p h+\sum_{i \in I_{+}} p_{i}\left(e_{i}+\delta e_{i}\right)+\sum_{i \in I_{\delta}} p_{i} e_{i}+\sum_{i \in I_{+}^{\prime}} p_{i}^{\prime}\left(e_{i}^{\prime}+\delta e_{i}^{\prime}\right)+\sum_{i \in I_{\delta}^{\prime}} p_{i}^{\prime} e_{i}^{\prime}+\sum_{i \in I_{+}^{\prime \prime}} p_{i}^{\prime \prime}\left(e_{i}^{\prime \prime}+\delta e_{i}^{\prime \prime}\right)
$$

for some non-negative $p, p_{i}, p_{i}^{\prime}, p_{i}^{\prime \prime}$ with at least one coefficient in each family $\left\{p_{i} \mid i \in I_{+} \sqcup I_{\delta}\right\},\left\{p_{i}^{\prime} \mid i \in I_{+}^{\prime} \sqcup I_{\delta}^{\prime}\right\}$, $\left\{p_{i}^{\prime \prime} \mid i \in I_{+}^{\prime \prime}\right\}$ being zero. In particular, in the symplectic case,

i) if $Q$ has one $\sigma$-fixed vertex and one $\sigma$-fixed arrow (i.e. $\left.Q=\widetilde{A}_{k, l}^{1,1}\right)$, then $p_{\frac{1}{2}+1}$ and $p_{1}^{\prime}$ have to be even,

ii) if $Q$ has one or two $\sigma$-fixed vertices and it has not any $\sigma$-fixed arrows (i.e. $Q=\widetilde{A}_{k, l}^{0,2}$ or $\left.\widetilde{D}_{n}^{0,1}\right)$, then both $p_{i}$ 's and $p_{j}^{\prime}$ 's, with $i \in I_{\delta}$ and $j \in I_{\delta}^{\prime}$, have to be even.

Proof. It follows by Lemma 3.6 and by decomposition of any regular dimension vector of the underlying quiver of $(Q, \sigma)$. In particular, since symplectic spaces with odd dimension don't exist, it implies $i)$ and $i i)$. 
Graphically we can represent, in the symmetric case, $\Delta$ (similarly $\Delta^{\prime}$ and $\left.\Delta^{\prime \prime}\right)$ as the polygons

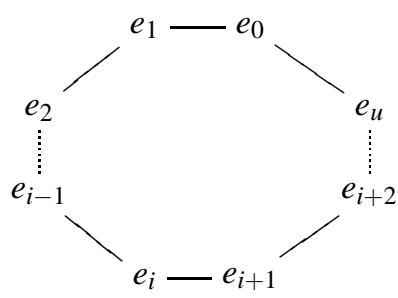

if $Q=\widetilde{A}_{k, k}^{0,0}$ and
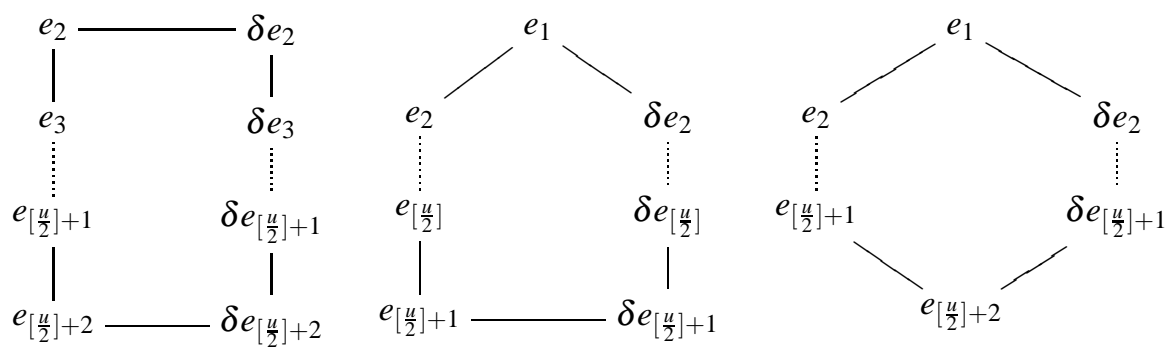

with a reflection respect to a central vertical line, in the other cases.

Definition 3.8. We define an involution $\sigma_{I}$ on the set of indices I such that $e_{\sigma_{I}(i)}=\delta e_{i}$ for every $i \in I$. Hence $\sigma_{I}(I)=I^{\prime}$ for $\widetilde{A}_{k, k}^{0,0}$ and $\sigma_{I} I_{+}=I_{-}, \sigma_{I} I_{\delta}=I_{\delta}$ for the other cases. Similarly we define an involution $\sigma_{I^{\prime}}$ and an involution $\sigma_{I^{\prime \prime}}$ respectively on $I^{\prime}$ and on $I^{\prime \prime}$.

In the remainder of the section, we shall call

$$
d^{\prime}=\sum_{i \in I_{+}} p_{i}\left(e_{i}+\delta e_{i}\right)+\sum_{i \in I_{\delta}} p_{i} e_{i}+\sum_{i \in I_{+}^{\prime}} p_{i}^{\prime}\left(e_{i}^{\prime}+\delta e_{i}^{\prime}\right)+\sum_{i \in I_{\delta}^{\prime}} p_{i}^{\prime} e_{i}^{\prime}+\sum_{i \in I_{+}^{\prime \prime}} p_{i}^{\prime \prime}\left(e_{i}^{\prime \prime}+\delta e_{i}^{\prime \prime}\right) .
$$

Proposition 3.9. If $d$ is regular with decomposition (14) such that $d=d^{\prime}$ or $d$ is not regular then $\operatorname{SpRep}(Q, d)$ (respectively $O R e p(Q, d)$ ) has an open $\operatorname{Sp}(Q, d)$-orbit (respectively $O(Q, d)$-orbit).

Proof. If $d=d^{\prime}$, we have no indecomposable of dimension vector $p h$ and so there are finitely many orbits. If $d$ is not regular, it follows from [19], Theorem 3.2.

In the next, $d$ shall be a regular symmetric dimension vector with decomposition (14) with $p \geq 1$ and $p \neq 0$. Now we shall describe the generators of $\operatorname{SpSI}(Q, d)$ and $\operatorname{SSI}(Q, d)$.

By Proposition 3.9. $S p\left(Q, d^{\prime}\right)$ acting on $\operatorname{SpRep}\left(Q, d^{\prime}\right)$ has an open orbit so, by Lemma 1.26, dimension of $\operatorname{SpSI}\left(Q, d^{\prime}\right)_{\chi^{\prime}}$ is 0 or 1 . This allows us to identify one non-zero element of $\operatorname{SpSI}(Q, d)_{\chi}$ with the element of $\operatorname{SpSI}(Q, p h)_{\chi}$ to which it restricts. Similarly one proceeds for $\operatorname{OSI}(Q, d)$.

We proceed now to describe the generators of the algebra $\operatorname{SpSI}(Q, d)$ (respectively $\operatorname{OSI}(Q, d)$ ). If the corresponding $I, I^{\prime}, I^{\prime \prime}$ are not empty, we label the vertices $e_{i}, e_{i}^{\prime}, e_{i}^{\prime \prime}$ of the polygons $\Delta, \Delta^{\prime}, \Delta^{\prime \prime}$ with the coefficients $p_{i}, p_{i}^{\prime}, p_{i}^{\prime \prime}$. We recall that

a) we have to label with $p_{i}$ (respectively with $p_{i}^{\prime}$ and $p_{i}^{\prime \prime}$ ) both vertices $e_{i}$ and $\delta e_{i}$, i.e $p_{i}=p_{\sigma_{I}(i)}$ (respectively $p_{i}^{\prime}=p_{\sigma_{I}^{\prime}(i)}^{\prime}$ and $\left.p_{i}^{\prime \prime}=p_{\sigma_{I}^{\prime \prime}(i)}^{\prime \prime}\right)$, if $e_{i} \neq \delta e_{i}$.

and in the symplectic case, by $i$ ) and ii) of Proposition 3.7

b) for $\widetilde{A}_{k, l}^{1,1}, p_{\frac{l}{2}+1}$ and $p_{1}^{\prime}$ have to be even,

c) for $\widetilde{A}_{k, l}^{0,2}$ and $\widetilde{D}_{n}^{0,1}, p_{i} \in I_{\delta}$ and $p_{i}^{\prime} \in I_{\delta}^{\prime}$ have to be even. 
We shall call these labelled polygons respectively $\Delta(d), \Delta^{\prime}(d), \Delta^{\prime \prime}(d)$.

Definition 3.10. We shall say that the labelled arc $p_{i}-\cdots . . . .-p_{j}$ (in clockwise orientation) of the labelled polygon $\Delta(d)$ is admissible if $p_{i}=p_{j}$ and $p_{i}<p_{k}$ for every its interior labels $p_{k}$. We denote such a labelled arc $p_{i}-\cdots \cdots \ldots \ldots \ldots$ - $p_{j}$ by $[i, j]$, and we define $p_{i}=p_{j}$ the index ind $[i, j]$ of $[i, j]$. Similarly we define admissible arcs and their indexes for the labelled polygons $\Delta^{\prime}(d)$ and $\Delta^{\prime \prime}(d)$.

We denote by $\mathscr{A}(d), \mathscr{A}^{\prime}(d), \mathscr{A}^{\prime \prime}(d)$ the sets of all admissible labelled arcs in the polygons $\Delta(d), \Delta^{\prime}(d)$, $\Delta^{\prime \prime}(d)$ respectively. In particular we note that if $d=p h$, then the polygons $\Delta(d), \Delta^{\prime}(d), \Delta^{\prime \prime}(d)$ are labelled by zeros and so $\mathscr{A}(d), \mathscr{A}^{\prime}(d), \mathscr{A}^{\prime \prime}(d)$ consist of all edges of respective polygons. With these notations we have the following

Proposition 3.11. For each arc $[i, j]$ from $\mathscr{A}(d)$ (respectively $\mathscr{A}^{\prime}(d)$ and $\mathscr{A}^{\prime \prime}(d)$ ) there exists in $\operatorname{SpSI}(Q, d)$ and in $\operatorname{OSI}(Q, d)$ a non zero semi-invariant

(i) of type $c^{E_{i-1, j}}$ (respectively $c^{E_{i-1, j}^{\prime}}$ and $c^{E_{i-1, j}^{\prime \prime}}$ ) or of type $c^{V_{(\varphi, \psi)}}$, with $(\varphi, \psi) \in\{(1,0),(0,1),(1,1)\}$;

(ii) of type $p f^{E_{i-1, j}}$ (respectively $p f^{E_{i-1, j}^{\prime}}$ and $p f^{E_{i-1, j}^{\prime \prime}}$ ) or of type $p f^{V_{(\varphi, \psi)}}$, with $(\varphi, \psi) \in\{(1,0),(0,1),(1,1)\}$, if $E_{i, j-1}, E_{i-1, j}^{\prime}, E_{i-1, j}^{\prime \prime}$ and $V_{(\varphi, \psi)}$ satisfy property $(O p)$ in the symplectic case and property (Spp) in the orthogonal case.

Let $c_{0}, \ldots, c_{t}$ be the coefficients of $\varphi^{t-i} \psi^{i}$ in $c^{V_{(\varphi, \psi)}}$ or $p f^{V_{(\varphi, \psi)}}$. We note case by case, from previous section, that $t$ can be $\frac{p-1}{2}, \frac{p}{2}$ or $p$. The generators of algebras $\operatorname{SpSI}(Q, d)$ and $\operatorname{SSI}(Q, d)$ are described by the following theorem

Theorem 3.12. Let $(Q, \sigma)$ a symmetric quiver of tame type and $d=p h+d^{\prime}$ the decomposition of a regular symmetric dimension vector $d$ with $p \geq 1$. Then $\operatorname{SpSI}(Q, d)($ respectively $\operatorname{OSI}(Q, d))$ is generated by

(i) $c_{0}, \ldots, c_{t}$;

(ii) $c^{E_{i-1, j}}, c^{E_{r-1, s}^{\prime},}, c^{E_{f-1, g}^{\prime \prime}}$ and $c^{V_{(\varphi, \psi)}}$ with $[i, j] \in \mathscr{A}(d),[r, s] \in \mathscr{A}^{\prime}(d),[f, g] \in \mathscr{A}^{\prime \prime}(d)$ and $(\varphi, \psi) \in\{(1,0),(0,1),(1,1)\}$;

(iii) $p f^{E_{i-1, j}}, p f^{E_{r-1, s}^{\prime}}, p f^{E_{f-1, g}^{\prime \prime}}$ and $p f^{V_{(\varphi, \psi)}}$ with $[i, j] \in \mathscr{A}(d),[r, s] \in \mathscr{A}^{\prime}(d),[f, g] \in \mathscr{A}^{\prime \prime}(d)$ and $(\varphi, \psi) \in$ $\{(1,0),(0,1),(1,1)\}$, if $E_{i-1, j}, E_{r-1, s}^{\prime}, E_{f-1, g}^{\prime \prime}$ and $V_{(\varphi, \psi)}$ satisfy property $(O p)$ (respectively property (Spp)).

For every regular dimension vector $d$, we note that $\langle h, d\rangle=0$ and

$$
\left\langle\underline{\operatorname{dim}} E_{i-1, j}, d\right\rangle=0 \Leftrightarrow p_{i}=p_{j} .
$$

So theorem 3.12 is equivalent to theorems 1.23 and 1.24

\subsubsection{Generic decomposition for symmetric quivers}

In the proof of theorem 3.12, we use the notion of generic decomposition of the symmetric dimension vector $d$ (see remark 2.8(a) in [12]).

Definition 3.13. A decomposition $\alpha=\beta_{1} \oplus \cdots \oplus \beta_{q}$ of a dimension vector $\alpha$ is called generic if there is a Zariski open subset $\mathscr{U}$ of $\operatorname{Re} p(Q, \alpha)$ such that each $U \in \mathscr{U}$ decomposes in $U=\bigoplus_{i=1}^{q} U_{i}$ with $U_{i}$ indecomposable representation of dimension $\beta_{i}$, for every $i \in\{1, \ldots, q\}$.

Definition 3.14. (1) A decomposition $\alpha=\beta_{1} \oplus \cdots \oplus \beta_{q}$ of a symmetric dimension vector $\alpha$ is called symplectic generic if there is a Zariski open subset $\mathscr{U}$ of $\operatorname{SpRep}(Q, \alpha)$ such that each $U \in \mathscr{U}$ decomposes in $U=\bigoplus_{i=1}^{q} U_{i}$ with $U_{i}$ indecomposable symplectic representation of dimension $\beta_{i}$, for every $i \in\{1, \ldots, q\}$.

(2) A decomposition $\alpha=\beta_{1} \oplus \cdots \oplus \beta_{q}$ of a symmetric dimension vector $\alpha$ is called orthogonal generic if there is a Zariski open subset $\mathscr{U}$ of ORep $(Q, \alpha)$ such that each $U \in \mathscr{U}$ decomposes in $U=\bigoplus_{i=1}^{q} U_{i}$ with $U_{i}$ indecomposable orthogonal representation of dimension $\beta_{i}$, for every $i \in\{1, \ldots, q\}$. 
For tame quivers the generic decomposition of any regular dimension vector is given by results of section 3 in [19].

We describe this decomposition explicitly for a symmetric regular dimension vector $d$ with decomposition (14). In the remainder of this section we set

$$
\begin{gathered}
\bar{d}=\sum_{i \in I_{+}} p_{i}\left(e_{i}+\delta e_{i}\right)+\sum_{i \in I_{\delta}} p_{i} e_{i}, \\
\bar{d}^{\prime}=\sum_{i \in I_{+}^{\prime}} p_{i}^{\prime}\left(e_{i}^{\prime}+\delta e_{i}^{\prime}\right)+\sum_{i \in I_{\delta}^{\prime}} p_{i}^{\prime} e_{i}^{\prime} \\
\bar{d}^{\prime \prime}=\sum_{i \in I_{+}^{\prime \prime}} p_{i}^{\prime \prime}\left(e_{i}^{\prime \prime}+\delta e_{i}^{\prime \prime}\right) .
\end{gathered}
$$

Remark 3.15. We can assume $p_{i}=0$ for $i \in I_{\delta}$ or $p_{i}=0$, for $i \in I_{+}$, and so $p_{\sigma_{I}(i)}=0$.

Definition 3.16. We divide the polygon $\Delta(\bar{d})$ in two parts:

(i) the up part $\Delta_{u p}(\bar{d})$ is the part of $\Delta(\bar{d})$ from $p_{i-1}$ to $p_{\sigma_{I}(i-1)}$;

(ii) the down part $\Delta_{\text {down }}(\bar{d})$ is the part of $\Delta(\bar{d})$ from $p_{\sigma_{I}(i+1)}$ to $p_{i+1}$.

Similarly for $\Delta^{\prime}$ and $\Delta^{\prime \prime}$.

Remark 3.17. We note that if $p_{i}=0$ with $i \in I_{\delta}$, then we have only the part $\Delta_{u p}$ or the part $\Delta_{\text {down }}$.

We consider $\Delta$, similarly one proceeds for $\Delta^{\prime}$ and $\Delta^{\prime \prime}$.

Definition 3.18. We shall call symmetric arc, an arc invariant under $\sigma_{I}$, i.e. an arc of type $\left[i, \sigma_{I}(i)\right]$.

Remark 3.19. By the division of $\Delta$ in $\Delta_{u p}$ and $\Delta_{\text {down }}$, we note that all symmetric arcs pass through the same $\sigma_{I}$-fixed vertex of $\Delta$ or through the same $\sigma_{I}$-fixed edge of $\Delta$.

Lemma 3.20. Let $(Q, \sigma)$ be a symmetric quiver of tame type.

(i) If $n=\sigma_{I}(n)$ then either there exists unique $x \in Q_{0}^{\sigma}$ such that $e_{n}(x) \neq 0$ or there exists unique a $\in Q_{1}^{\sigma}$ such that $e_{n}(t a) \neq 0$.

(ii) If $n-\sigma_{I}(n)$ is a $\sigma_{I}$-fixed edge in $\Delta$, then there exists unique $a \in Q_{1}^{\sigma}$ such that $e_{n}(t a) \neq 0$.

Proof. One proves type by type, using Lemma 3.6 (for further details, see Lemma 3.2.18 in [2]).

Definition 3.21. (i) If $n=\sigma_{I}(n)$, we call $x(n)$ the unique $x \in Q_{0}^{\sigma}$ such that $e_{n}(x) \neq 0$.

(ii) If $n=\sigma_{I}(n)$ or $n-\sigma_{I}(n)$ is a $\sigma_{I}$-fixed edge in $\Delta$, we call $a(n)$ the unique $a \in Q_{1}^{\sigma}$ such that $e_{n}(t a) \neq 0$.

Definition 3.22. For every $\operatorname{arc}[i, j]$ in $\Delta$, we define

$$
e_{[i, j]}=\sum_{k \in[i, j]} e_{k}
$$

Definition 3.23. (i) $\mathscr{A}_{+}(\bar{d}):=\left\{[i, j] \in \mathscr{A}(\bar{d}) \mid[i, j] \subset I_{+}\right\}$

(ii) $\mathscr{A}_{+}^{k}(\bar{d}):=\left\{[i, j] \in \mathscr{A}(\bar{d}) \mid[i, j] \subset I_{+}\right.$, ind $\left.[i, j]=k\right\}$.

(iii) $\mathscr{A}_{\sigma_{I}}^{k}(\bar{d})=\left\{[i, j]=\sigma_{I}[i, j] \in \mathscr{A}(\bar{d}) \mid\right.$ ind $\left.[i, j]=k\right\}$.

Remark 3.24. $[i, j] \subset I_{+}$if and only if $\left[\sigma_{I}(j), \sigma_{I}(i)\right] \subset I_{-}$and ind $[i, j]=\operatorname{ind}\left[\sigma_{I}(j), \sigma_{I}(i)\right]$. 
First we consider all the admissible $\operatorname{arcs}$ in $\mathscr{A}_{\sigma_{I}}^{r}(\bar{d}) \cup \mathscr{A}_{+}^{r}(\bar{d})$ such that $r=\max \left\{p_{k}\right\}$. So we get

$$
\begin{gathered}
\sum_{i \in I_{+}} p_{i}\left(e_{i}+\delta e_{i}\right)+\sum_{i \in I_{\delta}} p_{i} e_{i}= \\
\sum_{i \in I_{+}} \tilde{p}_{i}\left(\tilde{e}_{i}+\delta \tilde{e}_{i}\right)+\sum_{i \in I_{\delta}} \tilde{p}_{i} \tilde{e}_{i}+\left(\bigoplus_{[i, j] \in \mathscr{A}_{+}^{r}(\bar{d})}\left(e_{[i, j]}+\delta e_{[i, j]}\right)+\bigoplus_{\left[i, \sigma_{I}(i)\right] \in \mathscr{A}_{\sigma_{I}}^{r}(\bar{d})} e_{\left[i, \sigma_{I}(i)\right]}\right),
\end{gathered}
$$

where $\max \left(\tilde{p}_{i}\right)=r-1$. Then we repeat the procedure for (19) and so on we have

$$
\begin{gathered}
\sum_{i \in I_{+}} p_{i}\left(e_{i}+\delta e_{i}\right)+\sum_{i \in I_{\delta}} p_{i} e_{i}= \\
\bigoplus_{k=1}^{r}\left(\bigoplus_{[i, j] \in \mathscr{A}_{+}^{k}(\bar{d})}\left(e_{[i, j]}+\delta e_{[i, j]}\right)+\bigoplus_{\left[i, \sigma_{I}(i)\right] \in \mathscr{A}_{\sigma_{I}}^{k}(\bar{d})} e_{\left[i, \sigma_{I}(i)\right]}\right) .
\end{gathered}
$$

Remark 3.25. (i) If $[i, j]$ and $\left[i^{\prime}, j^{\prime}\right]$ are two admissible arcs in $\mathscr{A}(\bar{d})$ such that $[i, j] \supseteq\left[i^{\prime}, j^{\prime}\right]$, then ind $[i, j] \leq$ ind $\left[i^{\prime}, j^{\prime}\right]$.

(ii) If there not exists $[i, j] \in \mathscr{A}_{\sigma_{I}}^{h}(\bar{d}) \cup \mathscr{A}_{+}^{h}(\bar{d})$ such that $[i, j] \supseteq\left[i^{\prime}, j^{\prime}\right]$ for some $\left[i^{\prime}, j^{\prime}\right] \in \mathscr{A}_{\sigma_{I}}^{k}(\bar{d}) \cup \mathscr{A}_{+}^{k}(\bar{d})$, then the symmetric dimension vector corresponding to $\left[i^{\prime}, j^{\prime}\right]$ appears $k$-times in the decomposition (20), with $1 \leq h<k$.

Definition 3.26. Let $\left[i_{1}, j_{1}\right], \ldots,\left[i_{k}, j_{k}\right]$ be the admissible arcs such that $\left[i_{1}, j_{1}\right] \supseteq \cdots \supseteq\left[i_{k}, j_{k}\right]$, with $k \geq 1$. We define $q_{\left[i_{h}, j_{h}\right]}=\operatorname{ind}\left[i_{h}, j_{h}\right]-$ ind $\left[i_{h-1}, j_{h-1}\right]$ for every $1 \leq h \leq k$, where ind $\left[i_{0}, j_{0}\right]=0$.

We note that for every $[i, j] \in \mathscr{A}_{\sigma_{I}}^{k}(\bar{d}) \cup \mathscr{A}_{+}^{k}(\bar{d}), q_{[i, j]}$ is the multiplicity of the symmetric dimension vector corresponding to $[i, j]$ in the decomposition (20).

Finally we have

$$
\begin{gathered}
\sum_{i \in I_{+}} p_{i}\left(e_{i}+\delta e_{i}\right)+\sum_{i \in I_{\delta}} p_{i} e_{i}= \\
\bigoplus_{[i, j] \in \mathscr{A}_{+}(\bar{d})}\left(e_{[i, j]}+\delta e_{[i, j]}\right)^{\oplus q_{[i, j]}}+\bigoplus_{\left[i, \sigma_{I}(i)\right] \in \mathscr{A}(\bar{d})}\left(e_{\left[i, \sigma_{I}(i)\right]}\right)^{\oplus q_{\left[i, \sigma_{I}(i)\right]}} .
\end{gathered}
$$

Example 3.27. If $\Delta$ is of the form

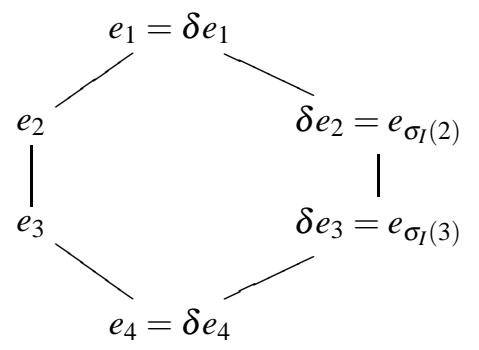

and $p_{1}=4, p_{2}=3, p_{3}=0$ and $p_{4}=2$, then $\left[2, \sigma_{I}(2)\right]=\left\{2,1, \sigma_{I}(2)\right\} \subset I_{+} \sqcup I_{\delta} \sqcup I_{-}$with $q_{\left[2, \sigma_{I}(2)\right]}=$ ind $\left[2, \sigma_{I}(2)\right]=$ $3,[1,1]=\{1\} \in I_{+}$with $q_{[1,1]}=$ ind $[1,1]-$ ind $\left[2, \sigma_{I}(2)\right]=1$ and $[4,4]=\{4\} \in I_{\delta}$ with $q_{[4,4]}=$ ind $[4,4]=2$. So we have

$$
\sum_{i \in I_{+}} p_{i}\left(e_{i}+\delta e_{i}\right)+\sum_{i \in I_{\delta}} p_{i} e_{i}=\left(\left(e_{2}+\delta e_{2}\right)+e_{1}\right)^{\oplus 3} \oplus e_{1} \oplus\left(e_{4}\right)^{\oplus 2} .
$$

Similarly we proceed with the decomposition of $\bar{d}^{\prime}$ and $\bar{d}^{\prime \prime}$. So we have the following 
Proposition 3.28. Let $(Q, \sigma)$ be a symmetric quiver of tame type and let $d$ be a symmetric dimension vector of a representation of the underlying quiver $Q$ with decomposition (14). Then

$$
\begin{array}{r}
d=\bigoplus_{i=1}^{p} h+\bigoplus_{[i, j] \in \mathscr{A}_{+}(\bar{d})}\left(e_{[i, j]}+\delta e_{[i, j]}\right)^{\oplus q_{[i, j]}+}+\bigoplus_{\left[i, \sigma_{I}(i)\right] \in \mathscr{A}(\bar{d})}\left(e_{\left[i, \sigma_{I}(i)\right]}\right)^{\oplus q_{\left[i, \sigma_{I}(i)\right]}}+ \\
\bigoplus_{[i, j] \in \mathscr{A}_{+}^{\prime}\left(\bar{d}^{\prime}\right)}\left(e_{[i, j]}^{\prime}+\delta e_{[i, j]}^{\prime}\right)^{\oplus q_{[i, j]}^{\prime}+} \bigoplus_{\left[i, \sigma_{I^{\prime}}(i)\right] \in \mathscr{A}^{\prime}\left(\bar{d}^{\prime}\right)}\left(e_{\left[i, \sigma_{I^{\prime}}(i)\right]}^{\prime}\right)^{\oplus q_{\left[i, \sigma_{I^{\prime}}^{\prime}(i)\right]}^{\prime}+} \\
\bigoplus_{[i, j] \in \mathscr{A}_{+}^{\prime \prime}\left(\bar{d}^{\prime \prime}\right)}\left(e_{[i, j]}^{\prime \prime}+\delta e_{[i, j]}^{\prime \prime}\right)^{\left.\oplus q_{[i, j]}^{\prime \prime}\right]}+\bigoplus_{\left[i, \sigma_{I^{\prime \prime}}(i)\right] \in \mathscr{A}^{\prime \prime}\left(\bar{d}^{\prime \prime}\right)}\left(e_{\left[i, \sigma_{I^{\prime \prime}}(i)\right]}^{\prime \prime}\right) q_{\left[i, \sigma_{I^{\prime \prime}}^{\prime \prime}(i)\right]}^{\prime \prime}
\end{array}
$$

is the generic decomposition of $d$.

We restrict to regular symplectic and orthogonal dimension vectors. We modify generic decomposition (23) of $d=\left(d_{i}\right)_{i \in Q_{0}}$ to get symplectic generic decomposition of $d$ or orthogonal generic decomposition of $d$.

Let $[i, j]$ be an arc in $\Delta_{u p}$ and let $[h, k]$ be an arc in $\Delta_{\text {down }}$. If $E_{[i, j]}$ is the regular indecomposable symplectic (respectively orthogonal) representation of $(Q, \sigma)$ corresponding to $[i, j]$ and $E_{[h, k]}$ is the regular indecomposable symplectic (respectively orthogonal) representation of $(Q, \sigma)$ corresponding to $[h, k]$, then

$$
\operatorname{Hom}_{Q}\left(E_{[i, j]}, E_{[h, k]}\right)=0=\operatorname{Hom}_{Q}\left(E_{[h, k]}, E_{[i, j]}\right)
$$

and

$$
\operatorname{Ext}_{Q}^{1}\left(E_{[i, j]}, E_{[h, k]}\right)=0=\operatorname{Ext}_{Q}^{1}\left(E_{[h, k]}, E_{[i, j]}\right) .
$$

So we deal separately with $\Delta_{u p}$ and $\Delta_{d o w n}$. We consider $I=I^{u p} \sqcup I^{\text {down }}, I_{+}=I_{+}^{u p} \sqcup I_{+}^{\text {down }}$ and $I_{\delta}=I_{\delta}^{u p} \sqcup I_{\delta}^{\text {down }}$. We have the decomposition $\bar{d}=\bar{d}_{\text {up }}+\bar{d}_{\text {down }}$, where

$$
\bar{d}_{u p}=\sum_{i \in I_{+}^{u p}} p_{i}\left(e_{i}+\delta e_{i}\right)+\sum_{i \in I_{\delta}^{u p}} p_{i} e_{i}
$$

and

$$
\bar{d}_{\text {down }}=\sum_{i \in I_{+}^{\text {down }}} p_{i}\left(e_{i}+\delta e_{i}\right)+\sum_{i \in I_{\delta}^{\text {down }}} p_{i} e_{i}
$$

By what has be said, the symplectic (respectively orthogonal) generic decomposition of $\bar{d}$ is direct sum of the symplectic (respectively orthogonal) generic decomposition of $\bar{d}_{u p}$ and the symplectic (respectively orthogonal) generic decomposition of $\bar{d}_{\text {down }}$.

Remark 3.29. (i) In the symplectic case, since $\bar{d}_{x}$ has to be even for every $x \in Q_{0}^{\sigma}$, we have to modify the symmetric dimension vectors corresponding to the arcs passing through the $\sigma_{I}$-fixed vertex $n$ such that there exists $x=x(n) \in Q_{0}^{\sigma}$.

(ii) In the orthogonal case, we have to modify the symmetric dimension vectors corresponding to the arcs passing through the $\sigma_{I}$-fixed vertex $n$ such that $\bar{d}_{t a(n)}$ is even and those corresponding to the arcs passing through the $\sigma_{I}$-fixed edge $n-\sigma_{I}(n)$ such that $\bar{d}_{t a(n)}$ is even.

(iii) We have to modify also ph $+e_{\left[i, \sigma_{I}(i)\right]}$, with $p$ odd, if $\left[i, \sigma_{I}(i)\right]$ is like in part (i) (respectively part (ii)), since $h+e_{\left[i, \sigma_{I}(i)\right]}$ is the dimension vector of regular indecomposable symplectic (respectively orthogonal) representation.

Definition 3.30. (i) $\mathscr{A}^{u p}(\bar{d})=\left\{[i, j] \in \mathscr{A}(\bar{d}) \mid[i, j] \subset I^{u p}\right\}$.

(ii) $\mathscr{A}_{+}^{u p}(\bar{d})=\left\{[i, j] \in \mathscr{A}(\bar{d}) \mid[i, j] \subset I_{+}^{u p}\right\}$. 
(iii) $\mathscr{A}^{\text {down }}(\bar{d})=\left\{[i, j] \in \mathscr{A}(\bar{d}) \mid[i, j] \subset I^{\text {down }}\right\}$.

(iv) $\mathscr{A}_{+}^{\text {down }}(\bar{d})=\left\{[i, j] \in \mathscr{A}(\bar{d}) \mid[i, j] \subset I_{+}^{\text {down }}\right\}$.

We restrict to $\Delta_{u p}$ (one proceeds similarly for $\Delta_{\text {down }}$ ). Let $\bar{d}=\bar{d}_{u p}+\bar{d}_{d o w n}$ be a regular symplectic dimension vector. $\Delta_{u p}$ contains either a $\sigma_{I}$-fixed vertex, we shall call $n_{u p}$, or a $\sigma_{I}$-fixed edge, we shall call $n_{u p}-\sigma_{I}\left(n_{u p}\right)$. Starting from generic decomposition (23) of $\bar{d}_{u p}$ we modify it as follows.

(1) We keep the summands $\left(e_{[i, j]}+\delta e_{[i, j]}\right)^{\oplus q_{[i, j]}}$ corresponding to the $\operatorname{arc}[i, j] \subset I_{+}^{u p}$.

(2) If $n_{u p}$ is such that there exists $a=a\left(n_{u p}\right) \in Q_{1}^{\sigma}$, then we keep the summands $\left(e_{\left[i, \sigma_{I}(i)\right]}\right)^{\oplus q_{\left[i, \sigma_{I}(i)\right]}}$ corresponding to the symmetric $\operatorname{arcs}\left[i, \sigma_{I}(i)\right]$ of $\Delta_{u p}$.

(3) If $n_{u p}$ is such that there exists $x=x\left(n_{u p}\right) \in Q_{0}^{\sigma}$, we have the symmetric dimension vectors

$$
e_{\left[i_{1}, \sigma_{I}\left(i_{1}\right)\right]}, \ldots, e_{\left[i_{2 s}, \sigma_{I}\left(i_{2 s}\right)\right]}
$$

corresponding to the $\operatorname{arcs}\left[i_{1}, \sigma_{I}\left(i_{1}\right)\right], \ldots,\left[i_{2 s}, \sigma_{I}\left(i_{2 s}\right)\right]$ such that $\left[i_{1}, \sigma_{I}\left(i_{1}\right)\right] \supseteq \cdots \supseteq\left[i_{2 s}, \sigma_{I}\left(i_{2 s}\right)\right]$. Then we divide them into pairs

$$
\left(\left[i_{2 k}, \sigma_{I}\left(i_{2 k}\right)\right],\left[i_{2 k-1}, \sigma_{I}\left(i_{2 k-1}\right)\right]\right),
$$

with $1 \leq k \leq s$. For each pair we consider $\left[i_{2 k}, \sigma_{I}\left(i_{2 k-1}\right)\right] \cup\left[i_{2 k-1}, \sigma_{I}\left(i_{2 k}\right)\right]$ and we substitute $e_{\left[i_{2 k}, \sigma_{I}\left(i_{2 k}\right)\right]} \oplus$ $e_{\left[i_{2 k-1}, \sigma_{I}\left(i_{2 k-1}\right)\right]}$ for

$$
e_{\left[i_{2 k}, \sigma_{I}\left(i_{2 k-1}\right)\right]}+e_{\left[i_{2 k-1}, \sigma_{I}\left(i_{2 k}\right)\right]} .
$$

So, by equation 21 in the symplectic case we get (see lemma 3.20 )

(i) if $n_{u p}$ is such that there exists $a=a\left(n_{u p}\right) \in Q_{1}^{\sigma}$,

$$
\bar{d}_{u p}=\bigoplus_{[i, j] \in \mathscr{A}_{+}^{u p}(\bar{d})}\left(e_{[i, j]}+\delta e_{[i, j]}\right)^{\oplus q_{[i, j]}}+\bigoplus_{\left[i, \sigma_{I}(i)\right] \in \mathscr{A}^{u p}(\bar{d})}\left(e_{\left[i, \sigma_{I}(i)\right]}\right)^{\oplus q_{\left[i, \sigma_{I}(i)\right]}}
$$

(ii) If $n_{u p}$ is such that there exists $x=x\left(n_{u p}\right) \in Q_{0}^{\sigma}$,

$$
\bar{d}_{u p}=\bigoplus_{[i, j] \in \mathscr{A}_{+}^{u p}(\bar{d})}\left(e_{[i, j]}+\delta e_{[i, j]}\right)^{\oplus q_{[i, j]}}+\bigoplus_{k=1}^{s}\left(e_{\left[i_{2 k}, \sigma_{I}\left(i_{2 k-1}\right)\right]}+e_{\left[i_{2 k-1}, \sigma_{I}\left(i_{2 k}\right)\right]}\right) .
$$

Finally we have to modify like in (3) the dimension vector $p h+e_{\left[i, \sigma_{I}(i)\right]}$ if $p$ is odd and $\left[i, \sigma_{I}(i)\right]$ passes through $n_{u p}$ such that there exists $x=x\left(n_{u p}\right) \in Q_{0}^{\sigma}$.

Example 3.31. Let $(Q, \sigma)$ be the symmetric quiver $\widetilde{A}_{0,6}^{1,1}$. We recall that $x_{\frac{l}{2}}=\sigma\left(x_{\frac{l}{2}}\right)$. $\Delta$ has the form (22). As in example 3.27 let $p_{1}=4, p_{2}=3, p_{3}=0$ and $p_{4}=2$. The $\sigma_{I}$-fixed vertex 4 is such that $e_{4}\left(x_{\frac{l}{2}}\right) \neq 0$. The only symmetric arc passing through 4 is $[4,4]$. Thus we substitute $\left(e_{4}\right)^{\oplus 2}$ for $e_{4}+e_{4}$. So, in the symplectic case we get

$$
\sum_{i \in I_{+}} p_{i}\left(e_{i}+\delta e_{i}\right)+\sum_{i \in I_{\delta}} p_{i} e_{i}=\left(\left(e_{2}+\delta e_{2}\right)+e_{1}\right)^{\oplus 3} \oplus e_{1} \oplus 2 e_{4} .
$$

Similarly we proceed with the decomposition of $\bar{d}^{\prime}$ and $\bar{d}^{\prime \prime}$.

Let $\bar{d}=\bar{d}_{u p}+\bar{d}_{d o w n}$ be a regular orthogonal dimension vector. Starting from generic decomposition (23) of $\bar{d}_{u p}$ we modify it as follows.

(1) We keep the summands $\left(e_{[i, j]}+\delta e_{[i, j]}\right)^{\oplus q_{[i, j]}}$ corresponding to the $\operatorname{arc}[i, j] \subset I_{+}^{u p}$.

(2) If $n_{u p}$ is such that there exists $a=a\left(n_{u p}\right) \in Q_{1}^{\sigma}$ such that $\bar{d}_{t a}$ is odd or $n_{u p}$ is such that there exist $x=$

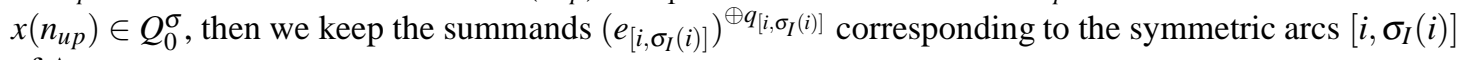
of $\Delta_{u p}$. 
(3) If $n_{u p}$ is such that there exists $a=a\left(n_{u p}\right) \in Q_{1}^{\sigma}$ such that $\bar{d}_{t a}$ is even, we have the symmetric dimension vectors

$$
e_{\left[i_{1}, \sigma_{I}\left(i_{1}\right)\right]}, \ldots, e_{\left[i_{2 s}, \sigma_{I}\left(i_{2 s}\right)\right]}
$$

corresponding to the $\operatorname{arcs}\left[i_{1}, \sigma_{I}\left(i_{1}\right)\right], \ldots,\left[i_{2 s}, \sigma_{I}\left(i_{2 s}\right)\right]$ such that $\left[i_{1}, \sigma_{I}\left(i_{1}\right)\right] \supseteq \cdots \supseteq\left[i_{2 s}, \sigma_{I}\left(i_{2 s}\right)\right]$. Then we divide them into pairs

$$
\left(\left[i_{2 k}, \sigma_{I}\left(i_{2 k}\right)\right],\left[i_{2 k-1}, \sigma_{I}\left(i_{2 k-1}\right)\right]\right),
$$

with $1 \leq k \leq s$. For each pair we consider $\left[i_{2 k}, \sigma_{I}\left(i_{2 k-1}\right)\right] \cup\left[i_{2 k-1}, \sigma_{I}\left(i_{2 k}\right)\right]$ and we substitute $e_{\left[i_{2 k}, \sigma_{I}\left(i_{2 k}\right)\right]} \oplus$ $e_{\left[i_{2 k-1}, \sigma_{I}\left(i_{2 k-1}\right)\right]}$ for

$$
e_{\left[i_{2 k}, \sigma_{I}\left(i_{2 k-1}\right)\right]}+e_{\left[i_{2 k-1}, \sigma_{I}\left(i_{2 k}\right)\right]} .
$$

So, by equation 21 in the orthogonal case we get

(i) if $n_{u p}$ is such that there exists $a=a\left(n_{u p}\right) \in Q_{1}^{\sigma}$ such that $\bar{d}_{t a}$ is odd or $n_{u p}$ is such that there exist $x=$ $x\left(n_{\text {up }}\right) \in Q_{0}^{\sigma}$,

$$
\bar{d}_{u p}=\bigoplus_{[i, j] \in \mathscr{A}_{+}^{u p}\left(d^{\prime}\right)}\left(e_{[i, j]}+\delta e_{[i, j]}\right)^{\oplus q_{[i, j]}}+\bigoplus_{\left[i, \sigma_{I}(i)\right] \in \mathscr{A}^{u p}\left(d^{\prime}\right)}\left(e_{\left[i, \sigma_{I}(i)\right]}\right)^{\oplus q_{\left[i, \sigma_{I}(i)\right]}}
$$

(ii) if $n_{u p}$ is such that there exists $a=a\left(n_{u p}\right) \in Q_{1}^{\sigma}$ such that $\bar{d}_{t a}$ is even,

$$
\bar{d}_{u p}=\bigoplus_{[i, j] \in \mathscr{A}_{+}^{u p}\left(d^{\prime}\right)}\left(e_{[i, j]}+\delta e_{[i, j]}\right)^{\oplus q_{[i, j]}}+\bigoplus_{k=1}^{s}\left(e_{\left[i_{2 k}, \sigma_{I}\left(i_{2 k-1}\right)\right]}+e_{\left[i_{2 k-1}, \sigma_{I}\left(i_{2 k}\right)\right]}\right) .
$$

Finally we have to modify like in (3) the dimension vector $p h+e_{\left[i, \sigma_{I}(i)\right]}$ if $p$ is odd and $\left[i, \sigma_{I}(i)\right]$ passes through $n_{u p}$ such that there exists $a=a\left(n_{u p}\right) \in Q_{1}^{\sigma}$ such that $\bar{d}_{t a}$ is even.

Example 3.32. Let $(Q, \sigma)$ be the symmetric quiver $\widetilde{A}_{0,6}^{1,1}$. We recall that $b=\sigma(b)$. $\Delta$ has the form (22).

As in example 3.27 let $p_{1}=4, p_{2}=3, p_{3}=0$ and $p_{4}=2$. The $\sigma_{I}$-fixed vertex 1 is such that $e_{1}(t b) \neq 0$ and $\bar{d}_{t b}$ is 2 . The only symmetric arcs passing through 1 is $\left[2, \sigma_{I}(2)\right]$. Thus we substitute $\left.\left(\left(e_{2}+\delta e_{2}\right)\right)+e_{1}\right)^{\oplus 3} \oplus e_{1}$ for $\left.\left.2\left(\left(e_{2}+\delta e_{2}\right)\right)+e_{1}\right) \oplus\left(\left(e_{2}+\delta e_{2}\right)\right)+2 e_{1}\right)$. So, in the orthogonal case we get

$$
\left.\sum_{i \in I_{+}} p_{i}\left(e_{i}+\delta e_{i}\right)+\sum_{i \in I_{\delta}} p_{i} e_{i}=2\left(\left(e_{2}+\delta e_{2}\right)+e_{1}\right) \oplus\left(\left(e_{2}+\delta e_{2}\right)\right)+2 e_{1}\right) \oplus\left(e_{4}\right)^{\oplus 2} .
$$

Similarly we proceed with the decomposition of $\bar{d}^{\prime}$ and $\bar{d}^{\prime \prime}$.

In general we have

Proposition 3.33. Let $(Q, \sigma)$ be a symmetric quiver of tame type.

(1) If $d$ is a regular symplectic dimension vector with decomposition (14). Then

$$
d=\bigoplus_{i=1}^{p} h \oplus \bar{d}_{u p} \oplus \bar{d}_{d o w n} \oplus \bar{d}_{u p}^{\prime} \oplus \bar{d}_{d o w n}^{\prime} \oplus \bar{d}_{u p}^{\prime \prime} \oplus \bar{d}_{d o w n}^{\prime \prime}
$$

is the symplectic generic decomposition of $d$.

(2) If $d$ is a regular orthogonal dimension vector with decomposition (14). Then

$$
d=\bigoplus_{i=1}^{p} h \oplus \bar{d}_{u p} \oplus \bar{d}_{\text {down }} \oplus \bar{d}_{u p}^{\prime} \oplus \bar{d}_{d o w n}^{\prime} \oplus \bar{d}_{u p}^{\prime \prime} \oplus \bar{d}_{d o w n}^{\prime \prime}
$$

is the orthogonal generic decomposition of $d$. 
For the proof, we need two propositions. We state these propositions only for regular indecomposable symplectic (respectively orthogonal) representations related to polygon $\Delta$, because for those related to polygon $\Delta^{\prime}$ and to polygon $\Delta^{\prime \prime}$ the statement and the proof are similar.

Proposition 3.34. Let $(Q, \sigma)$ be a symmetric quiver of tame tape. Let $V_{1} \neq V_{2}$ be two regular indecomposable symplectic (respectively orthogonal) representations of $(Q, \sigma)$ with symmetric dimension vector corresponding respectively to the arc $[i, j]$ and the arc $[h, k]$ of $\Delta\left(\Delta^{\prime}\right.$ or $\left.\Delta^{\prime \prime}\right)$. Moreover we suppose that $[i, j]$ and $[h, k]$ don't satisfy the following properties

(i) $[i, j] \cap[h, k] \neq \emptyset$ and $[i, j]$ doesn't contain $[h, k]$;

(ii) $[i, j] \cap[h, k] \neq \emptyset$ and $[h, k]$ doesn't contain $[i, j]$;

(iii) $[i, j]$ and $[h, k]$ are linked by one edge of $\Delta$ (respectively $\Delta^{\prime}$ or $\left.\Delta^{\prime \prime}\right)$.

Then $\operatorname{Ext}_{Q}^{1}\left(V_{1}, V_{2}\right)=0$.

Proof. We obtain the statement by

$$
\left\langle e_{i}, e_{j}\right\rangle= \begin{cases}1 & \text { if } i=j \\ -1 & \text { if } i=j-1 \\ 0 & \text { otherwise. }\end{cases}
$$

and applying Lemma 6.3 in chapter IX of [4] to the symplectic (respectively orthogonal) representations $V_{1}$ and $V_{2}$ corresponding to the arcs which don't satisfy (i), (ii) and (iii) (for more details, see Proposition 3.2.32 [2]).

Proposition 3.35. Let $(Q, \sigma)$ be a symmetric quiver of tame tape. Let $V$ be a regular indecomposable symplectic (respectively orthogonal) representation of $(Q, \sigma)$ such that $\underline{\operatorname{dim}}(V)=h$ or $\bar{d}$. Moreover we suppose $V \neq$ $E_{i, j} \oplus E_{\sigma_{I}(j), \sigma_{I}(i)}$ with $i, j \in I_{+}$such that $e_{i}(t a) \neq 0$ or $e_{j}(t a) \neq 0$ for $a \in Q_{1}^{\sigma}$. Then, for every non-trivial short exact sequence

$$
0 \rightarrow V \rightarrow W \rightarrow V \rightarrow 0
$$

$W$ is not symplectic (respectively it is not orthogonal).

Proof. The statement follows from an analysis case by case of symmetric quivers of tame type describing the non-trivial autoextension of each regular indecomposable symplectic (respectively orthogonal) $V$ of dimension $h$ or $\bar{d}$ such that $V \neq E_{i, j} \oplus E_{\sigma_{I}(j), \sigma_{I}(i)}$ with $i, j \in I_{+}$such that $e_{i}(t a) \neq 0$ or $e_{j}(t a) \neq 0$ for $a \in Q_{1}^{\sigma}$ (for details of the type $\widetilde{A}_{k, l}^{2,0,1}$, see Proposition 3.2.33 in [2]).

Proof of Proposition 3.33. (1) Let $d$ be a symplectic regular dimension vector with decomposition (30). First we note that the symmetric dimension vectors appearing in decomposition (30) are not dimension vectors of the regular indecomposable symplectic representations which are exceptions of proposition 3.34 and 3.35. Let $\mathscr{O}(d)$ be the open orbit of the regular symplectic representations of dimension $d$. By [Bo1] and [Z], we obtain each representation $V$ in $\mathscr{O}(d)$ as follows.

There are representations $M_{i}, U_{i}, V_{i}$ and short exact sequences

$$
0 \rightarrow U_{i} \rightarrow M_{i} \rightarrow V_{i} \rightarrow 0
$$

such that $M_{i+1}=U_{i} \oplus V_{i}$ and $V=U_{n+1} \oplus V_{n+1}$, with $1 \leq i \leq n$ for some $n \in \mathbb{N}$.

By Propositions 3.34 and 3.35, we have

(i) If $U_{i} \neq V_{i}$, then $\operatorname{Ext}_{Q}^{1}\left(V_{i}, U_{i}\right)=0$.

(ii) If $U_{i}=V_{i}$, then either $\operatorname{Ext}_{Q}^{1}\left(U_{i}, U_{i}\right)=0$ or no one non-trivial auto-extension of $U_{i}$ is symplectic. So, if $\operatorname{Ext}_{Q}^{1}\left(U_{i}, U_{i}\right) \neq 0$ then $U_{i}$ doesn't appear in decomposition of a symplectic representation.

Hence $V$ decomposes in regular indecomposable symplectic representations of dimension $\beta_{i}$, where $\beta_{i}$ are regular symmetric dimension vectors appearing in decomposition (30) of $d$.

(2) One proves similarly to (1). 


\subsubsection{Proof of Theorem 3.12}

By Proposition 2.4 in [3], Proposition 2.4 and Lemma 2.7, we can reduce the proof to the orientation of $\widetilde{A}$ as in proposition 2.4 and to the equiorientation for $\widetilde{D}$.

Let $d$ be a regular symmetric vector with a decomposition (30) or (31). We note that if $d=d_{1} \oplus d_{2}$ with $d_{1}$ and $d_{2}$ summands of this generic decomposition, we have canonical embeddings

$$
\operatorname{SpSI}(Q, d) \stackrel{\Phi_{d}}{\rightarrow} \bigoplus_{\chi \in \operatorname{char}(\operatorname{Sp}(Q, d))} \operatorname{SpSI}\left(Q, d_{1}\right)_{\left.\chi\right|_{d_{1}}} \otimes \operatorname{SpSI}\left(Q, d_{2}\right)_{\left.\chi\right|_{d_{2}}}
$$

and

$$
\operatorname{OSI}(Q, d) \stackrel{\Psi_{d}}{\rightarrow} \bigoplus_{\chi \in \operatorname{char}(O(Q, d))} \operatorname{OSI}\left(Q, d_{1}\right)_{\left.\chi\right|_{d_{1}}} \otimes \operatorname{OSI}\left(Q, d_{2}\right)_{\left.\chi\right|_{d_{2}}},
$$

induced by the restriction homomorphism. We prove theorem 3.12 by induction on the number of the summands $e_{[i, j]}+\delta e_{[i, j]}, e_{\left[i, \sigma_{I}(i)\right]}, e_{\left[i_{2 k}, \sigma_{I}\left(i_{2 k-1}\right)\right]}+e_{\left[i_{2 k-1}, \sigma_{I}\left(i_{2 k}\right)\right]}$ and respective summands corresponding to the admissible arcs in $\mathscr{A}^{\prime}(d)$ and in $\mathscr{A}^{\prime \prime}(d)$. If this number is 0 , then $d=p h$ and it was already proved. We suppose that the generic decomposition of $d$ contains one of those summands and, without loss of generality, we can assume that this summand is one of those corresponding to the arcs in $\mathscr{A}(d)$. In particular we suppose that this summand is $e_{\left[s, \sigma_{I}(s)\right]}$ (one proceeds similarly for the other types), with $s \in I_{+} \sqcup I_{\delta}$, and we can assume ind $\left[s, \sigma_{I}(s)\right]=r=\max \left\{p_{k}\right\}$. We call $d_{2}=e_{\left[s, \sigma_{I}(s)\right]}$ and so $d_{1}=d-e_{\left[s, \sigma_{I}(s)\right]}$. Now we compare the generators of the algebras $\operatorname{SpSI}(Q, d)$ and $\operatorname{SpSI}\left(Q, d_{1}\right)$ (respectively $\operatorname{SSI}(Q, d)$ and $\left.\operatorname{OSI}\left(Q, d_{1}\right)\right)$. By induction the generators of $\operatorname{SpSI}\left(Q, d_{1}\right)$ (respectively of $\left.\operatorname{SSI}\left(Q, d_{1}\right)\right)$ are described by theorem 3.12. Since $\Delta^{\prime}(d)=\Delta^{\prime}\left(d_{1}\right)$ and $\Delta^{\prime \prime}(d)=\Delta^{\prime \prime}\left(d_{1}\right)$, the generators $c_{0}, \ldots, c_{t}$ (with $t=\frac{p}{2}, \frac{p-1}{2}$ or $p$ ), those corresponding to the arcs from $\mathscr{A}^{\prime}(d)$ and those corresponding to the arcs from $\mathscr{A}^{\prime \prime}(d)$ occur. So it's enough to study the behavior of the semi-invariants corresponding to the arcs from $\mathscr{A}(d)$. We describe the link between the admissible arcs of the polygons $\Delta(d)$ and $\Delta\left(d_{1}\right)$. We have

$$
\begin{gathered}
d_{1}=p h+\sum_{i \in I_{+} \backslash\left(I_{+} \cap\left[s, \sigma_{I}(s)\right]\right)} p_{i}\left(e_{i}+\delta e_{i}\right)+\sum_{i \in I_{\delta} \backslash\left(I_{\delta} \cap\left[s, \sigma_{I}(s)\right]\right)} p_{i} e_{i}+ \\
\sum_{i \in I_{+} \cap\left[s, \sigma_{I}(s)\right]} p_{i}\left(e_{i}+\delta e_{i}\right)+\sum_{i \in I_{\delta} \cap\left[s, \sigma_{I}(s)\right]} p_{i} e_{i}+ \\
\sum_{i \in I_{+}^{\prime}} p_{i}^{\prime}\left(e_{i}^{\prime}+\delta e_{i}^{\prime}\right)+\sum_{i \in I_{\delta}^{\prime}} p_{i}^{\prime} e_{i}^{\prime}+\sum_{i \in I_{+}^{\prime \prime}} p_{i}^{\prime \prime}\left(e_{i}^{\prime \prime}+\delta e_{i}^{\prime \prime}\right) .
\end{gathered}
$$

We have two cases

(1) $p_{s+1}=p_{\sigma_{I}(s)-1}<r-1$ with $s+1 \in I_{+}$,

(2) $p_{s+1}=p_{\sigma_{I}(s)-1}=r-1$ with $s+1 \in I_{+}$.

in the case (1) the only difference between the structure of $\mathscr{A}(d)$ and $\mathscr{A}\left(d_{1}\right)$ is that the admissible arcs $[s, s-$ $1],[s-1, s-2], \ldots,\left[\sigma_{I}(s)+1, \sigma(s)\right]$ are of index $r$ in $\mathscr{A}(d)$ and of index $r-1$ in $\mathscr{A}\left(d_{1}\right)$. In the case (2) we have the admissible $\operatorname{arc}\left[s+1, \sigma_{I}(s)-1\right]$ of index $r-1$. The admissible arcs $[s, s-1],[s-1, s-2], \ldots,\left[\sigma_{I}(s)+1, \sigma_{I}(s)\right]$ are of index $s$ in $\mathscr{A}(d)$ and the admissible arcs $[s+1, s],[s, s-1], \ldots,\left[\sigma_{I}(s)+1, \sigma_{I}(s)\right],\left[\sigma_{I}(s), \sigma_{I}(s)-1\right]$ are of index $r-1$ in $\mathscr{A}\left(d_{1}\right)$.

Now we prove that the embeddings $\Phi_{d}$ and $\Psi_{d}$ are isomorphisms and this will be done in two steps. We describe the strategy for the symplectic case, similarly one proceeds for the orthogonal case. The first step is to show case by case that the semi-invariants corresponding to the admissible arcs $[i, j]$ are non zero $c^{V}$ for some $V \in \operatorname{Re} p(Q)$ and, if $V$ satisfies property $(O p)$, they are non zero $p f^{V}$. For the first step, we will deal only with the case $\widetilde{A}_{k, l}^{1,1}$, the other ones are similar. The second step is to give an explicit description of the generators of the algebras on the right hand side of $\Phi_{d}$ and $\Psi_{d}$. This is based on the knowledge, given by inductive hypothesis, of the algebra $\operatorname{SpSI}\left(Q, d_{1}\right)$. Moreover, by Proposition 3.9, we know that $\operatorname{SpSI}\left(Q, d_{2}\right)$ is a polynomial ring since $\operatorname{Sp}\left(Q, d_{2}\right)$ has an open orbit in $\operatorname{SpRep}\left(Q, d_{2}\right)$ (see Lemma 1.26). So we can describe explicitly the generators of the algebra 
$\operatorname{SpSI}\left(Q, d_{2}\right)$ and we note that they are determinants or pfaffians. At that point we know the generators of the algebras on the right hand side of $\Phi_{d}$ and $\Psi_{d}$. Now, using the fact that these are determinants or pfaffians, we prove that they actually are in $\operatorname{SpSI}(Q, d)$ and that the embeddings $\Phi_{d}$ and $\Psi_{d}$ are isomorphisms.

We will consider case by case the semi-invariants corresponding to each admissible arc $[i, j]$ for $\widetilde{A}_{k, l}^{1,1}$ (for the other symmetric quivers of tame type see section 3.2.1 in [2]). To simplify the notation we shall call $a$ both the arrow $a \in Q_{1}$ and the linear map $V(a)$ defined on $a$, where $V$ is a representation of $Q$.

We have at most two $C^{+}$-orbits $\Delta$ and $\Delta^{\prime}$ of the dimension vectors of nonhomogeneous simple regular representation. We assume $n \geq 2$ and we consider the $C^{+}$-orbit

$$
\left\{e_{1}=\delta e_{1}, e_{2}, \ldots, e_{\frac{l}{2}}, e_{\frac{l}{2}+1}=\delta e_{\frac{l}{2}+1}, \delta e_{\frac{l}{2}}, \ldots, \delta e_{2}\right\}
$$

Let $[i, j] \in \mathscr{A}(d)$. If we consider the arc $[1,1]$ of index 0 , i.e. $p_{1}=0, p_{2} \neq 0, \ldots, p_{\frac{l}{2}+1} \neq 0$, we have the minimal projective resolution of $V_{(0,1)}$

$$
0 \longrightarrow P_{\sigma\left(a_{0}\right)} \stackrel{d_{\text {min }}(0,1)}{\longrightarrow} P_{a_{0}} \longrightarrow V_{(0,1)} \longrightarrow 0
$$

where $d_{\min }^{V_{(0,1)}}=\sigma\left(v_{1}\right) \cdots \sigma\left(v_{\frac{l}{2}}\right) v_{\frac{l}{2}} \cdots v_{1}$ and so

$$
c^{V_{(0,1)}}=\operatorname{det}\left(\operatorname{Hom}_{Q}\left(d_{\min }^{V_{(0,1)}}, \cdot\right)\right)=\operatorname{det}\left(\sigma\left(v_{1}\right) \cdots \sigma\left(v_{\frac{l}{2}}\right) v_{\frac{l}{2}} \cdots v_{1}\right)
$$

in the orthogonal case and $p f^{V_{(0,1)}}=p f\left(\sigma\left(v_{1}\right) \cdots \sigma\left(v_{\frac{l}{2}}\right) v_{\frac{l}{2}} \cdots v_{1}\right)$ in the symplectic case, since by definition of symplectic representation $\sigma\left(v_{1}\right) \cdots \sigma\left(v_{\frac{l}{2}}\right) v_{\frac{l}{2}} \cdots v_{1}$ is skew-symmetric. If we consider the arc $\left[2, \sigma_{I}(2)\right]=[2,0]$ of index 0 , i.e. $p_{\sigma_{I}(2)}=$ $0=p_{2}, p_{1} \neq 0$, then we have the minimal projective resolution of $V_{(1,0)}$

$$
0 \longrightarrow P_{\sigma\left(a_{0}\right)} \stackrel{d_{\min }^{V_{(1,0)}}}{\longrightarrow} P_{a_{0}} \longrightarrow V_{(1,0)} \longrightarrow 0
$$

where $d_{\text {min }}^{V_{(1,0)}}=\sigma\left(u_{1}\right) \cdots \sigma\left(u_{\frac{k}{2}}\right) b u_{\frac{k}{2}} \cdots u_{1}$ and so

$$
c^{V_{(1,0)}}=\operatorname{det}\left(\operatorname{Hom}_{Q}\left(d_{\text {min }}^{V_{(1,0)}}, \cdot\right)\right)=\operatorname{det}\left(\sigma\left(u_{1}\right) \cdots \sigma\left(u_{\frac{k}{2}}\right) b u_{\frac{k}{2}} \cdots u_{1}\right)
$$

in the symplectic case and $p f^{V_{(1,0)}}=p f\left(\sigma\left(u_{1}\right) \cdots \sigma\left(u_{\frac{k}{2}}\right) b u_{\frac{k}{2}} \cdots u_{1}\right)$ in the orthogonal case, since $b$ is skewsymmetric and $\sigma\left(u_{i}\right)=-\left(u_{i}\right)^{t}$. We note that for $l=2$ we have only the admissible arcs $[1,1]$ an $\left[2, \sigma_{I}(2)\right]$. We assume now that $l \geq 4$ ( $l$ is even) and $[i, j]$ is not an admissible arc considered above. If $1 \leq j \leq i-1 \leq l$, then we identify $[i, j]$ with the path $v_{i-1} \cdots v_{j}$ in $Q$ and we have the minimal projective resolution of $E_{i-1, j}$

$$
0 \longrightarrow P_{x_{i-1}} \stackrel{d_{\min }^{E_{i-1, j}}}{\longrightarrow} P_{x_{j-1}} \longrightarrow E_{i-1, j} \longrightarrow 0
$$

where $d_{\min }^{E_{i-1, j}}=v_{i-1} \cdots v_{j}$ and so

$$
c^{E_{i-1, j}}=\operatorname{det}\left(\operatorname{Hom}_{Q}\left(d_{\min }^{E_{i-1, j}}, \cdot\right)\right)=\operatorname{det}\left(v_{i-1} \cdots v_{j}\right) .
$$

We note that

$$
c^{\tau^{-} \nabla E_{i-1, j}}=c^{E_{\sigma_{I}(j)-1, \sigma_{I}(i)}}=\operatorname{det}\left(\sigma\left(v_{j}\right) \cdots \sigma\left(v_{i-1}\right)\right)=\operatorname{det}\left(v_{i-1} \cdots v_{j}\right)=c^{E_{i-1, j}}
$$

Moreover, if $j=\sigma_{I}(i)$ then, only in the symplectic case, we get $p f\left(\sigma\left(v_{i}\right) \cdots v_{i}\right)=p f^{E_{i-1, \sigma_{I}(i)}}$ since $\sigma\left(v_{i}\right) \cdots v_{i}$ is skew-symmetric. Now we consider the arcs $[i, j]$ which have $e_{1}$ as internal vertex or as first vertex. For these arcs, $1 \leq i-1<j \leq l$ and we have the minimal projective resolution of $E_{i-1, j}$

$$
0 \longrightarrow P_{\sigma\left(a_{0}\right)} \oplus P_{x_{i-1}} \stackrel{d_{\min }^{E_{i-1, j}}}{\longrightarrow} P_{a_{0}} \oplus P_{x_{j-1}} \longrightarrow E_{i-1, j} \longrightarrow 0
$$


where $d_{\text {min }}^{E_{i-1, j}}=\left(\begin{array}{cc}\sigma\left(u_{1}\right) \cdots b \cdots u_{1} & \sigma\left(v_{1}\right) \cdots v_{j} \\ v_{i-1} \cdots v_{1} & 0\end{array}\right)$ and so

$$
c^{E_{i-1, j}}=\operatorname{det}\left(\operatorname{Hom}_{Q}\left(d_{\min }^{E_{i-1, j}}, \cdot\right)\right)=\operatorname{det}\left(\begin{array}{cc}
\sigma\left(u_{1}\right) \cdots b \cdots u_{1} & v_{i-1} \cdots v_{1} \\
\sigma\left(v_{1}\right) \cdots v_{j} & 0
\end{array}\right) .
$$

In particular we note that if $i=\sigma_{I}(j)$, in the orthogonal case, we get

$$
p f^{E_{\sigma_{I}(j)-1, j}}=p f\left(\begin{array}{cc}
\sigma\left(u_{1}\right) \cdots b \cdots u_{1} & v_{i-1} \cdots v_{1} \\
\sigma\left(v_{1}\right) \cdots \sigma\left(v_{i-1}\right) & 0
\end{array}\right),
$$

since $b$ is skew-symmetric, $\sigma\left(v_{i}\right)=-\left(v_{i}\right)^{t}$ and $\sigma\left(u_{i}\right)=-\left(u_{i}\right)^{t}$. Finally we note that $V_{(0,1)}, E_{i-1, \sigma_{I}(i)}$ satisfy $(O p)$ and $V_{(1,0)}, E_{\sigma_{I}(j)-1, j}$ satisfy property $(S p p)$. Similarly we define the semi-invariants for the admissible arcs $[i, j]$ in $\mathscr{A}^{\prime}(d)$, exchanging the upper paths of $\widetilde{A}_{k, l}^{1,1}$ with the lower ones.

We prove the second step of proof of Theorem 3.12. We note that if $[i, j]$ is admissible then the semi-invariants associated to $[i, j]$ define a nonzero element of $\operatorname{SpSI}(Q, d)$ (respectively of $\operatorname{OSI}(Q, d)$ ).

For a symmetric dimension vector $d$ we denote

$$
\operatorname{Sp} \Gamma(Q, d)=\left\{\chi \in \mathbb{Z}^{Q_{0}} \cup \frac{1}{2} \mathbb{Z}^{Q_{0}} \mid \operatorname{SpSI}(Q, d)_{\chi} \neq 0\right\}
$$

and

$$
O \Gamma(Q, d)=\left\{\chi \in \mathbb{Z}^{Q_{0}} \cup \frac{1}{2} \mathbb{Z}^{Q_{0}} \mid \operatorname{OSI}(Q, d)_{\chi} \neq 0\right\}
$$

the semigroup of weights of symplectic (respectively orthogonal) semi-invariants. We note that (34) and (35) involve also $\frac{1}{2} \mathbb{Z}^{Q_{0}}$ because in $\operatorname{SpSI}(Q, d)$ and in $\operatorname{OSI}(Q, d)$ also pfaffians can appear. To simplify the notation, we shall call $\chi_{[i, j]}, \chi_{[i, j]}^{\prime}$ and $\chi_{[i, j]}^{\prime \prime}$ be respectively the weights of the semi-invariants associated to admissible arcs $[i, j]$ respectively from $\mathscr{A}(d), \mathscr{A}^{\prime}(d)$ and $\mathscr{A}^{\prime \prime}(d)$. In the next the following proposition will be useful. We will state it only for $\Delta$, because for $\Delta^{\prime}$ and $\Delta^{\prime \prime}$ the statements are similar. Let $d$ be a regular symmetric dimension vector with decomposition $d=p h+d^{\prime}$ with $p \geq 1$.

Proposition 3.36. Let $(Q, \sigma)$ be a symmetric quiver of tame type. Let $d_{2}$ be of type $e_{\left[s, \sigma_{I}(s)\right]}, e_{[s, t]}+\delta e_{[s, t]}$ or $e_{\left[i_{2 k}, \sigma_{I}\left(i_{2 k-1}\right)\right]}+e_{\left[i_{2 k-1}, \sigma_{I}\left(i_{2 k}\right)\right]}$.

(i) If $d_{2}=e_{\left[s, \sigma_{I}(s)\right]}$, then

(a) For every arc $[i, j]$ of $\Delta^{\prime}$ and $\Delta^{\prime \prime}$ we have $\left.\chi_{[i, j]}^{\prime}\right|_{\operatorname{supp}\left(d_{2}\right)},\left.\chi_{[i, j]}^{\prime \prime}\right|_{\operatorname{supp}\left(d_{2}\right)} \in \operatorname{Sp} \Gamma\left(Q, d_{2}\right)\left(\right.$ respectively in $\left.O \Gamma\left(Q, d_{2}\right)\right)$.

(b) For every arc $[i, j]$ of $\Delta$ that doesn't intersect $\left[s, \sigma_{I}(s)\right]$ or contains $\left[s+1, \sigma_{I}(s)-1\right]$ we have $\left.\chi_{[i, j]}\right|_{\text {supp }\left(d_{2}\right)} \in$ $\operatorname{Sp} \Gamma\left(Q, d_{2}\right)$ (respectively in $O \Gamma\left(Q, d_{2}\right)$ ).

(c) Let $\rho_{1}, \ldots, \rho_{r}$ be the weights of generators of the polynomial algebra $\operatorname{SpSI}\left(Q, d_{2}\right)$ (respectively $O S I\left(Q, d_{2}\right)$ ). Then $r \geq n^{\prime}-s$, where $n^{\prime} \in I_{+} \sqcup I_{\delta}$ is either a $\sigma_{I}$-fixed vertex or the extremal vertex of a $\sigma_{I}$-fixed edge, and $\rho_{1}, \ldots, \rho_{r}$ can be reordered such that $\rho_{1}=\chi_{[s, s-1]}, \ldots, \rho_{n^{\prime}-s}=\chi_{\left[n^{\prime}+1, n^{\prime}\right]}$ and for every $m>n^{\prime}-s$ we have $\left\langle\rho_{m}, e_{n}\right\rangle=0$ for $n=s, \ldots, n^{\prime}$.

(ii) Let $d_{2}=e_{[s, t]}+\delta e_{[s, t]}$, then

(a) For every arc $[i, j]$ of $\Delta^{\prime}$ and $\Delta^{\prime \prime}$ we have $\left.\chi_{[i, j]}^{\prime}\right|_{\operatorname{supp}\left(d_{2}\right)},\left.\chi_{[i, j]}^{\prime \prime}\right|_{\operatorname{supp}\left(d_{2}\right)} \in \operatorname{Sp} \Gamma\left(Q, d_{2}\right)\left(\right.$ respectively in $\left.O \Gamma\left(Q, d_{2}\right)\right)$.

(b) For every symmetric arc $[i, j]$ of $\Delta$ that doesn't intersect $[s, t] \cup\left[\sigma_{I}(t), \sigma_{I}(s)\right]$ or contains $\left[s+1, \sigma_{I}(s+1)\right]$ or $\left[\sigma_{I}(t-1), t-1\right]$, we have $\left.\chi_{[i, j]}\right|_{\operatorname{supp}\left(d_{2}\right)} \in \operatorname{Sp} \Gamma\left(Q, d_{2}\right)$ (respectively in $\left.O \Gamma\left(Q, d_{2}\right)\right)$.

(c) For every arc $[i, j] \subset I_{+}\left(\right.$respectively $\left.[i, j] \subset I_{-}\right)$that doesn't intersect $[s, t]$ (respectively $\left.\left[\sigma_{I}(t), \sigma_{I}(s)\right]\right)$ or contains $[s+1, t-1]$ we have $\left.\chi_{[i, j]}\right|_{\text {supp }\left(d_{2}\right)} \in \operatorname{Sp} \Gamma\left(Q, d_{2}\right)$ (respectively in $O \Gamma\left(Q, d_{2}\right)$ ). 
(d) Let $\rho_{1}, \ldots, \rho_{r}$ be the weights of generators of the polynomial algebra $\operatorname{SpSI}\left(Q, d_{2}\right)$ (respectively $\left.O S I\left(Q, d_{2}\right)\right)$. Then $r \geq t-s$ and $\rho_{1}, \ldots, \rho_{r}$ can be reordered such that $\rho_{1}=\chi_{[s, s-1]}, \ldots, \rho_{t-s}=\chi_{[t+1, t]}$ and for every $m>t-s$ we have $\left\langle\rho_{m}, e_{n}\right\rangle=0$ for $n=s, \ldots, t$.

(iii) Let $d_{2}=e_{\left[i_{2 k}, i_{\sigma_{I}\left(i_{2 k-1}\right)}\right]}+e_{\left[i_{2 k-1}, i_{\sigma_{I}\left(i_{2 k}\right)}\right]}$, then

(a) For every arc $[i, j]$ of $\Delta^{\prime}$ and $\Delta^{\prime \prime}$ we have $\left.\chi_{[i, j]}^{\prime}\right|_{\operatorname{supp}\left(d_{2}\right)},\left.\chi_{[i, j]}^{\prime \prime}\right|_{\operatorname{supp}\left(d_{2}\right)} \in \operatorname{Sp} \Gamma\left(Q, d_{2}\right)\left(\right.$ respectively in $\left.O \Gamma\left(Q, d_{2}\right)\right)$.

(b) For every arc $[i, j]$ of $\Delta$ that doesn't intersect $\left[i_{2 k-1}, \sigma_{I}\left(i_{2 k-1}\right)\right]$ or contains $\left[i_{2 k-1}+1, \sigma_{I}\left(i_{2 k-1}\right)-1\right]$ we have $\left.\chi_{[i, j]}\right|_{\text {supp }\left(d_{2}\right)} \in \operatorname{Sp} \Gamma\left(Q, d_{2}\right)$ (respectively in $O \Gamma\left(Q, d_{2}\right)$ ).

(c) Let $\rho_{1}, \ldots, \rho_{r}$ be the weights of generators of the polynomial algebra $\operatorname{SpSI}\left(Q, d_{2}\right)$ (respectively $O S I\left(Q, d_{2}\right)$ ). Then $r \geq n^{\prime}-s$, where $n^{\prime} \in I_{+} \sqcup I_{\delta}$ is either a $\sigma_{I}$-fixed vertex or the extremal vertex of a $\sigma_{I}$-fixed edge, and $\rho_{1}, \ldots, \rho_{r}$ can be reordered such that $\rho_{1}=\chi_{[s, s-1]}, \ldots, \rho_{n^{\prime}-s}=\chi_{\left[n^{\prime}+1, n^{\prime}\right]}$ and for every $m>n^{\prime}-s$ we have $\left\langle\rho_{m}, e_{n}\right\rangle=0$ for $n=s, \ldots, n^{\prime}$.

Proof. It proceeds type by type analysis. We prove only the symplectic case for $Q=\widetilde{A}_{k, l}^{1,1}$ and for $d_{2}=e_{\left[s, \sigma_{I}(s)\right]}$, because the procedure to prove all other cases is similar. We order the vertices of $\widetilde{A}_{k, l}^{1,1}$ such that the only source is 1 (so the only sink is $\sigma(1)$ ), $h v_{i-1}=i$ for every $i \in\left\{2, \ldots, \frac{l}{2}+1\right\}, h u_{i}=\frac{l}{2}+i+1$ for every $i \in\left\{1, \ldots, \frac{k}{2}\right\}$ and then the respective conjugates by $\sigma$ of these. We shall call $w_{\left(t^{1}\right)_{i_{1}}, \ldots,\left(t^{f}\right)_{i_{f}}}$, where $t^{1}, \ldots, t^{f} \in \mathbb{Z} \cup \frac{1}{2} \mathbb{Z}$ and $\left\{i_{1}, \ldots, i_{f}\right\}$ is an ordered subset of $\left\{1, \ldots, \frac{l}{2}+\frac{k}{2}+1, \sigma\left(\frac{l}{2}+\frac{k}{2}+1\right)=\frac{l}{2}+\frac{k}{2}+2, \ldots, \sigma(1)=l+k+1\right\}$, the vector such that

$$
w_{\left(t^{1}\right)_{i_{1}}, \ldots,\left(t^{f}\right)_{i_{f}}}(y)= \begin{cases}\left(t^{j}\right)_{i_{j}} & y=i_{j}, \forall j=1, \ldots, f \\ 0 & \text { otherwise. }\end{cases}
$$

Moreover we can associate in bijective way the vertex $i \in\left\{2, \ldots, \frac{l}{2}\right\} \subset\left(\widetilde{A}_{k, l}^{1,1}\right)_{0}^{+}$to $i \in I_{+}$, the vertex $\frac{l}{2}+i+1$ of $\widetilde{A}_{k, l}^{1,1}$ to $i+1 \in I_{+}^{\prime}$ and the vertex $\frac{l}{2}+1$ to $\frac{l}{2}+1 \in I_{\delta}$.

(a) We have

$$
\chi_{[i, j]}^{\prime}=w_{(1)_{\frac{l}{2}+j},(-1)_{\frac{l}{2}+i}} \text { for } \quad 1 \leq j \leq i-1 \leq k+1,
$$

if $[i, j]$ has not $e_{1}$ as internal vertex;

$$
\chi_{[i, j]}^{\prime}=w_{(1)_{1},(-1)_{\frac{l}{2}+i},(1)_{\frac{l}{2}+j},(-1)_{\sigma(1)}} \quad \text { for } \quad 1 \leq i<j-1 \leq k+1
$$

if $[i, j]$ has $e_{1}$ as internal vertex and in particular if $j=\sigma_{I}(i)$ we have

$$
\chi_{[i, j]}^{\prime}=w_{\left(\frac{1}{2}\right)_{1},\left(-\frac{1}{2}\right)_{\frac{l}{2}+i},\left(\frac{1}{2}\right)_{\sigma\left(\frac{l}{2}+i\right)},\left(-\frac{1}{2}\right)_{\sigma(1)} .} .
$$

Now if $\left\langle\chi_{[i, j]}^{\prime}, e_{\left[s, \sigma_{I}(s)\right]}\right\rangle \neq 0$ then $\chi_{[i, j]}^{\prime} \notin S p S I\left(Q, d_{2}\right)$, but we note that $\left\langle\chi_{[i, j]}^{\prime}, e_{\left[s, \sigma_{I}(s)\right]}\right\rangle=0$ for every $i$ and $j$, so we have $(a)$.

(b) We have

$$
\chi_{[i, j]}=w_{(1)_{j},(-1)_{i}} \quad \text { for } \quad 1 \leq j \leq i-1 \leq l \quad \text { and } \quad \chi_{[\sigma(i), i]}=w_{\left(\frac{1}{2}\right)_{i},\left(-\frac{1}{2}\right)_{\sigma(i)}}
$$

if $[i, j]$ has not $e_{1}$ as internal vertex or as first vertex;

$$
\chi_{[i, j]}=w_{(1)_{1},(-1)_{i},(1)_{j},(-1)_{\sigma(1)}} \text { for } \quad 1 \leq i-1<j \leq l
$$

if $[i, j]$ has $e_{1}$ as internal vertex or as first vertex.

Now we note that $\left\langle\chi_{[i, j]}, e_{\left[s, \sigma_{I}(s)\right]}\right\rangle \neq 0$ if $[i, j] \cap[s, t] \neq \emptyset$ and $[i, j] \nsupseteq[s-1, \sigma(s+1)=\sigma(s)-1]$, so we have $(b)$. (c) First we note that we can choose symmetric arcs of each length from a fixed vertex of $\Delta$, because the result of Theorem 3.12 is invariant respect to the Coxeter transformation $\tau^{+}$. The generators of $\operatorname{SpSI}\left(Q, d_{2}\right)$ associated to $\Delta\left(d_{2}\right)$ are $c^{E_{i}}=\operatorname{det}\left(v_{i}\right)$ of weight $\chi_{[i, i+1]}=w_{(1)_{i},(-1)_{i+1}}$ for every $i \in\{1, \ldots, s-1\}$ and $c^{E_{s-1, \sigma_{I}(s)}}=$ 
$\operatorname{det}\left(\begin{array}{cc}\sigma\left(u_{1}\right) \cdots b \cdots u_{1} & v_{s} \cdots v_{1} \\ \sigma\left(v_{1}\right) \cdots \sigma\left(v_{s}\right) & 0\end{array}\right)$ of weight $\chi_{\left[s, \sigma_{I}(s)\right]}=w_{(1)_{1},(-1)_{s},(1)_{\sigma(s)},(-1)_{\sigma(1)}}$. So we call $\rho_{i}=\chi_{[i, i+1]}$ for every $i \in\{1, \ldots, s-1\}$ and $\rho_{n^{\prime}-s}=\chi_{\left[s, \sigma_{I}(s)\right]}$, where in this case $n^{\prime}=\frac{l}{2}+1$. The other generators are associated to $\Delta^{\prime}\left(d_{2}\right)$ and so, as done in the part $(a)$ of this proposition, their weight $\rho_{m}$, for $m \in\left\{n^{\prime}-s+1, \ldots, r\right\}$, are such that $\left\langle\rho_{m}, e_{n}\right\rangle=0$ for $n \in\left\{s, \ldots, n^{\prime}\right\}$.

We assume now that $d=d_{1}+d_{2}$ where $d_{1}=p h+d_{1}^{\prime}$ with $p \geq 1$ and $d_{2}=e_{\left[s, \sigma_{I}(s)\right]}, e_{[s, t]}+\delta e_{s, t]}$ or $e_{\left[i_{2 k}, i_{\sigma_{I}\left(i_{2 k-1}\right)}\right]}+$ $e_{\left[i_{2 k-1}, i_{\sigma_{I}\left(i_{2 k}\right]}\right]}$. So we take the corresponding arc in a chosen position (for which we proved proposition 3.36).

Proposition 3.37. Let $d, d_{1}, d_{2}$ be as above. We suppose that the semigroup $\operatorname{Sp} \Gamma\left(Q, d_{1}\right)\left(\right.$ respectively $\left.O \Gamma\left(Q, d_{1}\right)\right)$ is generated by the weights $\chi_{[i, j]}, \chi_{[i, j]}^{\prime}, \chi_{[i, j]}^{\prime \prime}$ for admissible arcs $[i, j]$ of the labelled polygons $\Delta\left(d_{1}\right), \Delta^{\prime}\left(d_{1}\right)$, $\Delta^{\prime \prime}\left(d_{1}\right)$. Then $\operatorname{Sp} \Gamma\left(Q, d_{1}\right) \cap \operatorname{Sp} \Gamma\left(Q, d_{2}\right)$ (respectively $\left.O \Gamma\left(Q, d_{1}\right) \cap O \Gamma\left(Q, d_{2}\right)\right)$ is generated by the weights $\chi_{[i, j]}$, $\chi_{[i, j]}^{\prime}, \chi_{[i, j]}^{\prime \prime}$ for admissible arcs $[i, j]$ of the labelled polygons $\Delta(d), \Delta^{\prime}(d), \Delta^{\prime \prime}(d)$.

Proof. We prove it only for the othogonal case and for $d_{2}=e_{\left[s, \sigma_{I}(s)\right]}$, because for the symplectic case e the other types of $d_{2}$, the proof is similar.

We are two cases.

(1) Assume $p_{s+1}=p_{\sigma_{I}(s)-1}<r-1$. The admissible arcs of $\Delta\left(d_{1}\right), \Delta^{\prime}\left(d_{1}\right), \Delta^{\prime \prime}\left(d_{1}\right)$ and $\Delta(d), \Delta^{\prime}(d), \Delta^{\prime \prime}(d)$ are the same. By Proposition $3.36 O \Gamma\left(Q, d_{2}\right)$ contains $\chi_{[s, s-1]}, \ldots, \chi_{\left[\sigma_{I}(s)+1, \sigma_{I}(s)\right]}$ and all the other weights corresponding to the admissible arcs of $\Delta(d), \Delta^{\prime}(d)$ and $\Delta^{\prime \prime}(d)$.

(2) Assume $p_{s+1}=p_{\sigma_{I}(s)-1}=r-1$. We prove that $O \Gamma\left(Q, d_{1}\right) \cap O \Gamma\left(Q, d_{2}\right)$ is generated by $\chi_{[i, j]}^{\prime}$ for every admissible arc $[i, j]$ of $\Delta^{\prime}\left(d_{1}\right)=\Delta^{\prime}(d), \chi_{[i, j]}^{\prime \prime}$ for every admissible arc $[i, j]$ of $\Delta^{\prime \prime}\left(d_{1}\right)=\Delta^{\prime \prime}(d)$ and $\chi_{[i, j]}$ for every admissible arc $[i, j]$ of $\Delta\left(d_{1}\right)$ of index smaller than $r-1$ or not intersecting $\left[s, \sigma_{I}(s)\right]$, i.e. $\chi_{[s, s-1]}, \ldots, \chi_{\left[\sigma_{I}(s)+1, \sigma_{I}(s)\right]}$ and $\chi_{\left[s+1, \sigma_{I}(s)-1\right]}=\chi_{[s+1, s]}+\cdots+\chi_{\left[\sigma_{I}(s), \sigma_{I}(s)-1\right]}$. Let

$$
\chi=\sum_{[i, j] \in \mathscr{A}\left(d_{1}\right)} n_{i, j} \chi_{[i, j]}+\sum_{[i, j] \in \mathscr{A}^{\prime}\left(d_{1}\right)} n_{i, j}^{\prime} \chi_{[i, j]}^{\prime}+\sum_{[i, j] \in \mathscr{A}^{\prime \prime}\left(d_{1}\right)} n_{i, j}^{\prime \prime} \chi_{[i, j]}^{\prime \prime},
$$

with $n_{i, j}, n_{i, j}^{\prime}, n_{i, j}^{\prime \prime} \geq 0$, be an element of $O \Gamma\left(Q, d_{1}\right)$. We assume that $\chi$ is also in $O \Gamma\left(Q, d_{2}\right)$. By Proposition 3.36 we note that all the generators of $O \Gamma\left(Q, d_{1}\right)$ except of $\chi_{[s+1, s]}$ and $\chi_{\left[\sigma_{I}(s), \sigma_{I}(s)-1\right]}$ are also in $O \Gamma\left(Q, d_{2}\right)$. Hence, if $\chi$ contains neither $\chi_{[s+1, s]}$ nor $\chi_{\left[\sigma_{I}(s), \sigma_{I}(s)-1\right]}$, then $\chi$ is a linear combination of desired generators. So we have to prove that if $\chi$ contains $\chi_{[s+1, s]}\left(\right.$ resp. $\left.\chi_{\left[\sigma_{I}(s), \sigma_{I}(s)-1\right]}\right)$ with positive coefficient, then it contains $\chi_{[s, s-1]}, \ldots, \chi_{\left[\sigma_{I}(s), \sigma_{I}(s)-1\right]}\left(\right.$ resp. $\left.\chi_{[s+1, s]}, \ldots, \chi_{\left[\sigma_{I}(s)+1, \sigma_{I}(s)\right]}\right)$. Thus we can subtract $\chi_{\left[s+1, \sigma_{I}(s)-1\right]}$ from $\chi$.

We assume that $\chi$ contains $\chi_{[s+1, s]}$ with positive coefficient (the proof is similar for $\left.\chi_{\left[\sigma_{I}(s), \sigma_{I}(s)-1\right]}\right)$. We note that $\left\langle\chi_{[s+1, s]}, e_{s}\right\rangle=1$ and, by Proposition 3.36 the other generators of $O \Gamma\left(Q, d_{1}\right)$, except $\chi_{[s, s-1]}$, have zero product scalar with $e_{s}$. Moreover, $\chi \in O \Gamma\left(Q, d_{2}\right)$ and so, by Proposition 3.36, $\left\langle\chi, e_{s}\right\rangle \leq 0$. Hence $\chi$ contains $\chi_{[s, s-1]}$ with positive coefficient. By Proposition 3.36, it follows that $\left\langle\chi, e_{s}+e_{s-1}\right\rangle \leq 0$. But $\left\langle\chi_{[s+1, s]}+\chi_{[s, s-1]}, e_{s}+e_{s-1}\right\rangle=1$ and $\chi_{[s-1, s-2]}$ is the only generator of $O \Gamma\left(Q, d_{1}\right)$ with negative scalar product with $e_{s}+e_{s-1}$. Continuing in this way, we check that $\chi$ contains $\chi_{[s+1, s]}, \chi_{[s, s-1]}, \ldots, \chi_{\left[\sigma_{I}(s)+1, \sigma_{I}(s)\right]}, \chi_{\left[\sigma_{I}(s), \sigma_{I}(s)-1\right]}$ with positive coefficients. So we can subtract $\chi_{\left[s+1 . \sigma_{I}(s)-1\right]}$ from $\chi$ and continue. In this way we complete the proof.

Now we can finish the proof of Theorem 3.12 Since Theorem 3.12 is equivalent to Theorems 1.23 and 1.24 for tame type and regular dimension vectors, then, in this way, we finish also the proof of theorems 1.23 and 1.24

Again we consider the embeddings

$$
\operatorname{SpSI}(Q, d) \stackrel{\Phi_{d}}{\rightarrow} \bigoplus_{\chi \in \operatorname{char}(\operatorname{Sp}(Q, d))} \operatorname{SpSI}\left(Q, d_{1}\right)_{\left.\chi\right|_{d_{1}}} \otimes \operatorname{SpSI}\left(Q, d_{2}\right)_{\left.\chi\right|_{d_{2}}}
$$

and

$$
\operatorname{OSI}(Q, d) \stackrel{\Psi_{d}}{\rightarrow} \bigoplus_{\chi \in \operatorname{char}(O(Q, d))} \operatorname{OSI}\left(Q, d_{1}\right)_{\left.\chi\right|_{d_{1}}} \otimes \operatorname{OSI}\left(Q, d_{2}\right)_{\left.\chi\right|_{d_{2}}}
$$


where $Q, d, d_{1}$ and $d_{2}$ are as above. The semigroup of weights of the right hand side of $\Phi_{d}$ and $\Psi_{d}$ are respectively $\operatorname{Sp} \Gamma\left(Q, d_{1}\right) \cap S p \Gamma\left(Q, d_{2}\right)$ and $O \Gamma\left(Q, d_{1}\right) \cap O \Gamma\left(Q, d_{2}\right)$. These are generated by $\chi_{[i, j]}, \chi_{[i, j]}^{\prime}, \chi_{[i, j]}^{\prime \prime}$ for admissible arcs $[i, j]$ of the labelled polygons $\Delta(d), \Delta^{\prime}(d), \Delta^{\prime \prime}(d)$, by proposition 3.37 So the algebras on the right hand side of $\Phi_{d}$ and $\Psi_{d}$ are generated by the semi-invariants of weights $\chi_{[i, j]}, \chi_{[i, j]}^{\prime}, \chi_{[i, j]}^{\prime \prime}$ and by the semiinvariants of weights $\langle h, \cdot\rangle$ ( or $\frac{1}{2}\langle h, \cdot\rangle$ ).

Finally, we note that the embeddings $\Phi_{d}$ and $\Psi_{d}$ are isomorphisms because they are also isomorphisms in the weight $\langle h, \cdot\rangle$ (or $\left.\frac{1}{2}\langle h, \cdot\rangle\right)$ and so we completed the proof of Theorem 3.12. Moreover, in that way, we also proved Proposition 3.11] expliciting the semi-invariants of type $c^{V}$ for every admissible arc $[i, j]$, and the following theorem

Theorem 3.38. Let $(Q, \sigma)$ be a symmetric quiver of tame type and let $d$ be a regular symmetric dimension vector. We consider decomposition (14) with $p \geq 1$ and $d^{\prime} \neq 0$. There exist isomorphisms of algebras

$$
\operatorname{SpSI}(Q, d) \stackrel{\Phi_{d}}{\rightarrow} \bigoplus_{\chi \in \operatorname{char}(\operatorname{Sp}(Q, d))} \operatorname{SpSI}(Q, p h)_{\chi} \otimes \operatorname{SpSI}\left(Q, d^{\prime}\right)_{\chi^{\prime}}
$$

and

$$
\operatorname{OSI}(Q, d) \stackrel{\Psi_{d}}{\rightarrow} \bigoplus_{\chi \in \operatorname{char}(O(Q, d))} \operatorname{OSI}(Q, p h)_{\chi} \otimes \operatorname{OSI}\left(Q, d^{\prime}\right)_{\chi^{\prime}}
$$

where $\chi^{\prime}=\left.\chi\right|_{d^{\prime}}$, i.e. the restriction of the weight $\chi$ to the support of $d^{\prime}$.

\section{References}

[1] K. Akin, D. Buchsbaum, J. Weyman, Schur functors and Schur complexes, Advances Math. 44 (1982), 207-277.

[2] R. Aragona, Semi-invariants of symmetric quivers, $\mathrm{PhD}$ thesis, arXiv:1006.4378v1 [math.RT], (2009).

[3] R. Aragona, Semi-invariants of symmetric quivers of finite type, preprint, arXiv:0910.1070v3 [math.RT], (2009).

[4] I. Assem, D. Simson, A. Skowronski, Elements of the Representation Theory of Associative Algebras, volume 1, London Mathematical Society Students Texts 65, Cambridge University Press, 2006.

[5] I. N. Bernstein, I. M. Gelfand, V. A. Ponomarev, Coxeter functors and Gabriel's theorem, Uspekhi Mat. Nauk 28, no. 2(170) (1973), 19-33.

[6] V. Dlab, C. M. Ringel, Indecomposable Representations of Graphs and Algebras, Memoirs Amer. Math. Soc. 173 (1976).

[7] H. Derksen, J. Weyman, Semi-invariants of quivers and saturation for Littlewood-Richardson coefficients, J. Amer. Math. Soc. 16 (2000),467-479.

[8] H. Derksen, J. Weyman, Generalized quivers associated to reductive groups, Colloq. Math. 94 (2002), No. $2,151-173$.

[9] M. Domokos, A.N. Zubkov, Semi-invariants of quivers as determinants, Trans- form. Groups, 6 no. 1, (2001), 9-24.

[10] W. Fulton, J. Harris, Representation Theory; the first course, Graduate Texts in Mathematics 129, SpringerVerlag, 1991.

[11] K. Igusa, K. Orr, G. Todorov, and J. Weyman, Cluster complexes via semi-invariants, Compos. Math. 145 (2009), no. 4, 1001-1034. 
[12] V. G. Kac, Infinite root systems representations of graphs and invariant theory, Invent. Math. 56 (1980), 57-92.

[13] A. A. Lopatin, Invariants of quivers under the action of classical groups, J. Algebra 321 (2009), no. 4, 1079-1106.

[14] A. A. Lopatin, A. N. Zubkov, Semi-invariants of mixed representations of quivers, Transform. Groups 12 (2007), no. 2, 341-369.

[15] I. G. Macdonald, Symmetric functions and Hall polynomials, second edition, with contributions by A. Zelevinsky, Oxford Mathematical Monographs, Oxford University Press, New York, 1995.

[16] P. Magyar, J. Weyman and A. Zelevinsky, Multiple flag varieties of finite type, Adv. Math. 141 (1999), no. $1,97-118$.

[17] C. Procesi, Lie Groups. An Approach through Invariants and Representations, Universitext, Springer, New York, 2007.

[18] C. M, Ringel, Representations of K-species and bimodules, J. Algebra 41 (1976), 269-302.

[19] C.M., Ringel, The rational invariants of the tame quivers, Invent. Math. 58 (1980), 217-239.

[20] A. Schofield, Semi-invariants of quivers, J. London Math. Soc. (3) 65 (1992), 46-64.

[21] A. Schofield, M. Van den Bergh, Semi-invariants of quivers for arbitrary di- mension vectors, Indag. Math. (N.S.) 12 no. 1, (2001), 125-138.

[22] D.A. Shmelkin, Signed quivers, symmetric quivers, and root systems, J. Lond. Math. Soc., II. Ser. 73, no. 3, (2006), 586-606.

[23] M. Sato, T. Kimura, A classification of irreducible prehomogeneous vector spaces and their relative invariants, Nagoya J. Math 65 (1977), 1-155.

[24] A.N. Zubkov, Invariants of mixed representations of quivers I, J. Algebra Appl., 4 no. 3, (2005), 245-285. 